\title{
On the Lagrangian Structure of Integrable Hierarchies
}

\author{
Yuri B. Suris and Mats Vermeeren
}

\begin{abstract}
We develop the concept of pluri-Lagrangian structures for integrable hierarchies. This is a continuous counterpart of the pluri-Lagrangian (or Lagrangian multiform) theory of integrable lattice systems. We derive the multi-time Euler Lagrange equations in their full generality for hierarchies of two-dimensional systems, and construct a pluri-Lagrangian formulation of the potential Korteweg-de Vries hierarchy.
\end{abstract}

\section{Introduction}

In this paper, our departure point are two developments which have taken place in the field of discrete integrable systems in recent years.

- Firstly, multi-dimensional consistency of lattice systems has been proposed as a notion of integrability $[8,15]$. In retrospect, this notion can be seen as a discrete counterpart of the well-known fact that integrable systems never appear alone but are organized into integrable hierarchies. Based on the notion of multi-dimensional consistency, a classification of two-dimensional integrable lattice systems (the so called ABS list) was given in [1]. Moreover, for all equations of the ABS list, considered as equations on $\mathbb{Z}^{2}$, a variational interpretation was found in [1].

- Secondly, the idea of the multi-dimensional consistency was blended with the variational formulation in [13], where it was shown that solutions of any ABS equation on any quad surface $\Sigma$ in $\mathbb{Z}^{N}$ are critical points of a certain action functional $\int_{\Sigma} \mathscr{L}$ obtained by integration of a suitable discrete Lagrangian two-form $\mathscr{L}$. Moreover, it was observed in [13] that the critical value of the action remains invariant under local changes of the underlying quad-surface, or, in other words, that the 2-form $\mathscr{L}$ is closed on solutions of quad-equations, and it was suggested to consider this as a defining feature of integrability. However, later research [10] revealed that

Y.B. Suris $(\bowtie) \cdot M$. Vermeeren

Inst. für Mathematik, Technische Universität Berlin, Straße des 17. Juni 136,

10623 Berlin, Germany

e-mail: suris@math.tu-berlin.de

M. Vermeeren

e-mail: vermeer@math.tu-berlin.de

(C) The Author(s) 2016

A.I. Bobenko (ed.), Advances in Discrete Differential Geometry,

DOI 10.1007/978-3-662-50447-5_11 
$\mathscr{L}$ is closed not only on solutions of (non-variational) quad-equations, but also on general solutions of the corresponding Euler-Lagrange equations. Therefore, at least for discrete systems, the closedness condition is implicitly contained in the variational formulation.

A general theory of multi-time one-dimensional Lagrangian systems, both discrete and continuous, has been developed in [20]. A first attempt to formulate the theory for continuous two-dimensional systems was made in [21]. For such systems, a solution is a critical point of the action functional $\int_{S} \mathscr{L}$ on any two-dimensional surface $S$ in $\mathbb{R}^{N}$, where $\mathscr{L}$ is a suitable differential two-form. The treatment in [21] was restricted to second order Lagrangians, i.e. to two-forms $\mathscr{L}$ that only depend on the second jet bundle. In the present work we will extend this to Lagrangians of any order.

As argued in [10], the unconventional idea to consider the action on arbitrary two-dimensional surfaces in the multi-dimensional space of independent variables has significant precursors. These include:

- Theory of pluriharmonic functions and, more generally, of pluriharmonic maps $[11,17,19]$. By definition, a pluriharmonic function of several complex variables $f: \mathbb{C}^{N} \rightarrow \mathbb{R}$ minimizes the Dirichlet functional $E_{\Gamma}=\int_{\Gamma}\left|(f \circ \Gamma)_{z}\right|^{2} d z \wedge d \bar{z}$ along any holomorphic curve in its domain $\Gamma: \mathbb{C} \rightarrow \mathbb{C}^{N}$. Differential equations governing pluriharmonic functions,

$$
\frac{\partial^{2} f}{\partial z_{i} \partial \bar{z}_{j}}=0 \quad \text { for all } \quad i, j=1, \ldots, N,
$$

are heavily overdetermined. Therefore it is not surprising that pluriharmonic functions (and maps) belong to the theory of integrable systems.

This motivates the term pluri-Lagrangian systems, which was proposed in $[9,10]$.

- Baxter's Z-invariance of solvable models of statistical mechanics [3, 4]. This concept is based on invariance of the partition functions of solvable models under elementary local transformations of the underlying planar graphs. It is well known (see, e.g., [7]) that one can identify planar graphs underlying these models with quad-surfaces in $\mathbb{Z}^{N}$. On the other hand, the classical mechanical analogue of the partition function is the action functional. This suggests the relation of $\mathrm{Z}$ invariance to the concept of closedness of the Lagrangian 2-form, at least at the heuristic level. This relation has been made mathematically precise for a number of models, through the quasiclassical limit [5, 6].

- The classical notion of variational symmetry, going back to the seminal work of E. Noether [16], has been shown to be directly related to the closedness of the Lagrangian form in the multi-time [21].

The main goal of this paper is two-fold: to derive the Euler Lagrange equations for two-dimensional pluri-Lagrangian problems of arbitrary order, and to state the (potential) $\mathrm{KdV}$ hierarchy as a pluri-Lagrangian system. We will also discuss the closedness of the Lagrangian two-form, which turns out to be related to the Hamiltonian theory of integrable hierarchies. 
Note that the influential monograph [12], according to the foreword, is "about hierarchies of integrable equations rather than about individual equations". However, its Lagrangian part (Chaps. 19, 20) only deals with individual equations. The reason for this is apparently the absence of the concept of pluri-Lagrangian systems. We hope that this paper opens up the way for a variational approach to integrable hierarchies.

\section{Pluri-Lagrangian Systems}

\subsection{Definition}

We place our discussion in the formalism of the variational bicomplex as presented in [12, Chap. 19] (and summarized, for the reader's convenience, in Appendix A). Slightly different versions of this theory can be found in [18] and in [2].

Consider a vector bundle $X: \mathbb{R}^{N} \rightarrow \mathbb{R}$ and its $n$th jet bundle $J^{n} X$. Let $\mathscr{L} \in$ $\mathscr{A}^{(0, d)}\left(J^{n} X\right)$ be a smooth horizontal $d$-form. In other words, $\mathscr{L}$ is a $d$-form on $\mathbb{R}^{N}$ whose coefficients depend on a function $u: \mathbb{R}^{N} \rightarrow \mathbb{R}$ and its partial derivatives up to order $n$. We call $\mathbb{R}^{N}$ the multi-time, $u$ the field, and $\mathscr{L}$ the Lagrangian $d$-form. We will use coordinates $\left(t_{1}, \ldots, t_{N}\right)$ on $\mathbb{R}^{N}$. Recall that in the standard calculus of variations the Lagrangian is a volume form, so that $d=N$.

Definition 2.1 We say that the field $u$ solves the pluri-Lagrangian problem for $\mathscr{L}$ if $u$ is a critical point of the action $\int_{S} \mathscr{L}$ simultaneously for all $d$-dimensional surfaces $S$ in $\mathbb{R}^{N}$. The equations describing this condition are called the multi-time EulerLagrange equations. We say that they form a pluri-Lagrangian system and that $\mathscr{L}$ is a pluri-Lagrangian structure for these equations.

To discuss critical points of a pluri-Lagrangian problem, consider the vertical derivative $\delta \mathscr{L}$ of the $(0, d)$-form $\mathscr{L}$ in the variational bicomplex, and a variation $\mathscr{V}$. Note that we consider variations $\mathscr{V}$ as vertical vector fields; such a restriction is justified by our interest, in the present paper, in autonomous systems only. Besides, in the context of discrete systems only vertical vector fields seem to possess a natural analogs. The criticality condition of the action, $\delta \int_{S} \mathscr{L}=0$, is described by the equation

$$
\int_{S} \iota_{\operatorname{pr} \mathscr{V}} \delta \mathscr{L}=0,
$$

which has to be satisfied for any variation $\mathscr{V}$ on $S$ that vanishes at the boundary $\partial S$. Recall that $\operatorname{pr} \mathscr{V}$ is the $n$th jet prolongation of the vertical vector field $\mathscr{V}$, and that $\iota$ stands for the contraction. One fundamental property of critical points can be established right from the outset.

Proposition 2.2 The exterior derivative $\mathrm{d} \mathscr{L}$ of the Lagrangian is constant on critical points $u$. 
Proof Consider a critical point $u$ and a small $(d+1)$-dimensional ball $B$. Because $S:=\partial B$ has no boundary, Eq. (1) is satisfied for any variation $\mathscr{V}$. Using Stokes' theorem and the properties that $\delta \mathrm{d}+\mathrm{d} \delta=0$ and $\iota_{\mathrm{pr} \mathscr{V}} \mathrm{d}+\mathrm{d} \iota_{\mathrm{pr} \mathscr{V}}=0$ (Propositions A. 1 and A.4 in Appendix A), we find that

$$
0=\int_{\partial B} \iota_{\operatorname{pr} \mathscr{V}} \delta \mathscr{L}=\int_{B} \mathrm{~d}\left(\iota_{\operatorname{pr} \mathscr{V}} \delta \mathscr{L}\right)=-\int_{B} \iota_{\operatorname{pr} \mathscr{V}} \mathrm{d}(\delta \mathscr{L})=\int_{B} \iota_{\operatorname{pr} \mathscr{V}} \delta(\mathrm{d} \mathscr{L}) .
$$

Since this holds for any ball $B$ it follows that $\iota_{\operatorname{pr} \mathscr{V}} \delta(\mathrm{d} \mathscr{L})=0$ for any variation $\mathscr{V}$ of a critical point $u$. Therefore, $\delta(\mathrm{d} \mathscr{L})=0$, so that $\mathrm{d} \mathscr{L}$ is constant on critical points $u$. Note that here we silently assume that the space of critical points is connected. It would be difficult to justify this property in any generality, but it is usually clear in applications, where the critical points are solutions of certain well-posed systems of partial differential equations.

We will take a closer look at the property $\mathrm{d} \mathscr{L}=$ const in Sect. 6 , when we discuss the link with Hamiltonian theory. It will be shown that vanishing of this constant, i.e., closedness of $\mathscr{L}$ on critical points, is related to integrability of the multi-time Euler-Lagrange equations.

\subsection{Approximation by Stepped Surfaces}

For computations, we will use the multi-index notation for partial derivatives. For any multi-index $I=\left(i_{1}, \ldots, i_{N}\right)$ we set

$$
u_{I}=\frac{\partial^{|I|} u}{\left(\partial t_{1}\right)^{i_{1}} \ldots\left(\partial t_{N}\right)^{i_{N}}},
$$

where $|I|=i_{1}+\ldots+i_{N}$. The notations $I k$ and $I k^{\alpha}$ will represent the multi-indices $\left(i_{1}, \ldots, i_{k}+1, \ldots i_{N}\right)$ and $\left(i_{1}, \ldots, i_{k}+\alpha, \ldots i_{N}\right)$ respectively. When convenient we will also use the notations $I t_{k}$ and $I t_{k}^{\alpha}$ for these multi-indices. We will write $k \notin I$ if $i_{k}=0$ and $k \in I$ if $i_{k}>0$. We will denote by $\mathrm{D}_{i}$ or $\mathrm{D}_{t_{i}}$ the total derivative with respect to coordinate direction $t_{i}$,

$$
\mathrm{D}_{i}:=\mathrm{D}_{t_{i}}:=\sum_{I} u_{I i} \frac{\partial}{\partial u_{I}}
$$

and by $\mathrm{D}_{I}:=\mathrm{D}_{t_{1}}^{i_{1}} \ldots \mathrm{D}_{t_{N}}^{i_{N}}$ the corresponding higher order derivatives.

Our main general result is the derivation of the multi-time Euler-Lagrange equations for two-dimensional surfaces $(d=2)$. That will allow us to study the $\mathrm{KdV}$ hierarchy as a pluri-Lagrangian system. However, it is instructive to first derive the multi-time Euler-Lagrange equations for curves $(d=1)$. 
The key technical result used to derive multi-time Euler-Lagrange equations is the observation that it suffices to consider a very specific type of surface.

Definition 2.3 A stepped $d$-surface is a $d$-surface that is a finite union of coordinate $d$-surfaces. A coordinate $d$-surface of the direction $\left(i_{1}, \ldots, i_{d}\right)$ is a $d$-surface lying in an affine $d$-plane $\left\{\left(t_{1}, \ldots, t_{N}\right) \mid t_{j}=c_{j}\right.$ for $\left.j \neq i_{1}, \ldots, i_{d}\right\}$.

Lemma 2.4 If the action is stationary on any stepped surface, then it is stationary on any smooth surface.

The proof of this Lemma can be found in appendix B.

\subsection{Multi-time Euler-Lagrange Equations for Curves}

Theorem 2.5 Consider a Lagrangian 1-form $\mathscr{L}=\sum_{i=1}^{N} L_{i} \mathrm{~d} t_{i}$. The multi-time Euler-Lagrange equations for curves are:

$$
\begin{aligned}
& \frac{\delta_{i} L_{i}}{\delta u_{I}}=0 \quad \forall I \not \nexists i, \\
& \frac{\delta_{i} L_{i}}{\delta u_{I i}}=\frac{\delta_{j} L_{j}}{\delta u_{I j}} \quad \forall I,
\end{aligned}
$$

where $i$ and $j$ are distinct, and the following notation is used for the variational derivative corresponding to the coordinate direction $i$ :

$$
\frac{\delta_{i} L_{i}}{\delta u_{I}}=\sum_{\alpha \geq 0}(-1)^{\alpha} \mathrm{D}_{i}^{\alpha} \frac{\partial L_{i}}{\partial u_{I i^{\alpha}}}=\frac{\partial L_{i}}{\partial u_{I}}-\mathrm{D}_{i} \frac{\partial L_{i}}{\partial u_{I i}}+\mathrm{D}_{i}^{2} \frac{\partial L_{i}}{\partial u_{I i^{2}}}-\ldots
$$

Remark 2.6 In the special case that $\mathscr{L}$ only depends on the first jet bundle, system (2)-(3) reduces to the equations found in [20]:

$$
\begin{aligned}
& \frac{\delta_{i} L_{i}}{\delta u}=0 \quad \Leftrightarrow \quad \frac{\partial L_{i}}{\partial u}-\mathrm{D}_{i} \frac{\partial L_{i}}{\partial u_{i}}=0, \\
& \frac{\delta_{i} L_{i}}{\delta u_{j}}=0 \quad \Leftrightarrow \quad \frac{\partial L_{i}}{\partial u_{j}}=0 \text { for } i \neq j, \\
& \frac{\delta_{i} L_{i}}{\delta u_{i}}=\frac{\delta_{j} L_{j}}{\delta u_{j}} \quad \Leftrightarrow \quad \frac{\partial L_{i}}{\partial u_{i}}=\frac{\partial L_{j}}{\partial u_{j}} \text { for } i \neq j .
\end{aligned}
$$

Proof (of Theorem 2.5) According to Lemma 2.4, it is sufficient to look at a general L-shaped curve $S=S_{i} \cup S_{j}$, where $S_{i}$ is a line segment of the coordinate direction $i$ and $S_{j}$ is a line segment of the coordinate direction $j$. Denote the cusp by $p:=$ $S_{i} \cap S_{j}$. We orient the curve such that $S_{i}$ induces the positive orientation on the point $p$ and $S_{j}$ the negative orientation. There are four cases, depending on how 


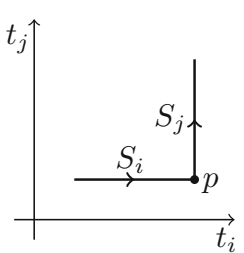

$(+1,+1)$

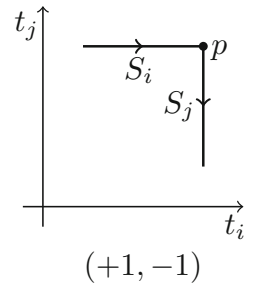

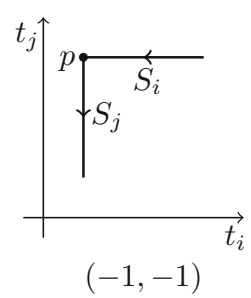

Fig. 1 The four L-shaped curves with their values of $\left(\varepsilon_{i}, \varepsilon_{j}\right)$

the $L$-shape is rotated. They are depicted in Fig. 1 . To each case we associate a pair $\left(\varepsilon_{i}, \varepsilon_{j}\right) \in\{-1,+1\}^{2}$, where the positive value is taken if the respective piece of curve is oriented in the coordinate direction, and negative if it is oriented opposite to the coordinate direction.

The variation of the action is

$$
\begin{aligned}
\int_{S} \iota_{\mathrm{pr} \mathscr{V}} \delta \mathscr{L} & =\varepsilon_{i} \int_{S_{i}}\left(\iota_{\mathrm{pr} \mathscr{V}} \delta L_{i}\right) \mathrm{d} t_{i}+\varepsilon_{j} \int_{S_{j}}\left(\iota_{\mathrm{pr} \mathscr{V}} \delta L_{j}\right) \mathrm{d} t_{j} \\
& =\varepsilon_{i} \int_{S_{i}} \sum_{I} \frac{\partial L_{i}}{\partial u_{I}} \delta u_{I}(\mathscr{V}) \mathrm{d} t_{i}+\varepsilon_{j} \int_{S_{j}} \sum_{I} \frac{\partial L_{j}}{\partial u_{I}} \delta u_{I}(\mathscr{V}) \mathrm{d} t_{j} .
\end{aligned}
$$

Note that these sums are actually finite. Indeed, since $\mathscr{L}$ depends on the $n$th jet bundle all terms with $|I|:=i_{1}+\ldots+i_{N}>n$ vanish.

Now we expand the sum in the first of the integrals and perform integration by parts.

$$
\begin{aligned}
& \varepsilon_{i} \int_{S_{i}}\left(\iota_{\operatorname{pr} \mathscr{V}} \delta L_{i}\right) \mathrm{d} t_{i} \\
& =\varepsilon_{i} \int_{S_{i}} \sum_{I \not \ngtr i}\left(\frac{\partial L_{i}}{\partial u_{I}} \delta u_{I}(\mathscr{V})+\frac{\partial L_{i}}{\partial u_{I i}} \delta u_{I i}(\mathscr{V})+\frac{\partial L_{i}}{\partial u_{I i^{2}}} \delta u_{I i^{2}}(\mathscr{V})+\ldots\right) \mathrm{d} t_{i} \\
& =\varepsilon_{i} \int_{S_{i}} \sum_{I \not \ngtr i}\left(\frac{\partial L_{i}}{\partial u_{I}}-\mathrm{D}_{i} \frac{\partial L_{i}}{\partial u_{I i}}+\mathrm{D}_{i}^{2} \frac{\partial L_{i}}{\partial u_{I i^{2}}}-\mathrm{D}_{i}^{3} \frac{\partial L_{i}}{\partial u_{I i^{3}}}+\ldots\right) \delta u_{I}(\mathscr{V}) \mathrm{d} t_{i} \\
& \quad+\sum_{I \not p i}\left(\frac{\partial L_{i}}{\partial u_{I i}} \delta u_{I}(\mathscr{V})+\frac{\partial L_{i}}{\partial u_{I i^{2}}} \delta u_{I i}(\mathscr{V})-\mathrm{D}_{i} \frac{\partial L_{i}}{\partial u_{I i^{2}}} \delta u_{I}(\mathscr{V})\right. \\
& \left.\quad+\frac{\partial L_{i}}{\partial u_{I i^{3}}} \delta u_{I i^{2}}(\mathscr{V})-\mathrm{D}_{i} \frac{\partial L_{i}}{\partial u_{I i^{3}}}(\mathscr{V}) \delta u_{I i}(\mathscr{V})+\mathrm{D}_{i}^{2} \frac{\partial L_{i}}{\partial u_{I i^{3}}} \delta u_{I}(\mathscr{V})+\ldots\right)\left.\right|_{p}
\end{aligned}
$$

Using the language of variational derivatives, this reads

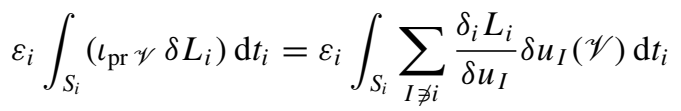




$$
\begin{gathered}
+\left.\sum_{I \not \ngtr i}\left(\frac{\delta_{i} L_{i}}{\delta u_{I i}} \delta u_{I}(\mathscr{V})+\frac{\delta_{i} L_{i}}{\delta u_{I i^{2}}} \delta u_{I i}(\mathscr{V})+\ldots\right)\right|_{p} \\
=\varepsilon_{i} \int_{S_{i}} \sum_{I \not \ngtr i} \frac{\delta_{i} L_{i}}{\delta u_{I}} \delta u_{I}(\mathscr{V}) \mathrm{d} t_{i}+\left.\sum_{I}\left(\frac{\delta_{i} L_{i}}{\delta u_{I i}} \delta u_{I}(\mathscr{V})\right)\right|_{p}
\end{gathered}
$$

The other piece, $S_{j}$, contributes

$$
\varepsilon_{j} \int_{S_{j}} \iota_{\mathrm{pr} \mathscr{V}} \delta L_{j} \mathrm{~d} t_{j}=\varepsilon_{j} \int_{S_{j}} \sum_{I \not j} \frac{\delta_{j} L_{j}}{\delta u_{I}} \delta u_{I}(\mathscr{V}) \mathrm{d} t_{j}-\left.\sum_{I}\left(\frac{\delta_{j} L_{j}}{\delta u_{I j}} \delta u_{I}(\mathscr{V})\right)\right|_{p},
$$

where the minus sign comes from the fact that $S_{j}$ induces negative orientation on the point $p$. Summing the two contributions, we find

$$
\begin{aligned}
\int_{S} \iota_{\operatorname{pr} \mathscr{V}} \delta \mathscr{L}= & \varepsilon_{i} \int_{S_{i}} \sum_{I \not \supset i} \frac{\delta_{i} L_{i}}{\delta u_{I}} \delta u_{I}(\mathscr{V}) \mathrm{d} t_{i}+\varepsilon_{j} \int_{S_{j}} \sum_{I \not \supset j} \frac{\delta_{j} L_{j}}{\delta u_{I}} \delta u_{I}(\mathscr{V}) \mathrm{d} t_{j} \\
& +\left.\sum_{I}\left(\frac{\delta_{i} L_{i}}{\delta u_{I i}} \delta u_{I}(\mathscr{V})-\frac{\delta_{j} L_{j}}{\delta u_{I j}} \delta u_{I}(\mathscr{V})\right)\right|_{p}
\end{aligned}
$$

Now require that the variation (4) of the action is zero for any variation $\mathscr{V}$. If we consider variations that vanish on $S_{j}$, then we find for every multi-index $I$ which does not contain $i$ that

$$
\frac{\delta_{i} L_{i}}{\delta u_{I}}=0
$$

Given this equation, and its analogue for the index $j$, only the last term remains in the right hand side of Eq. (4). Considering variations around the cusp $p$ we find for every multi-index $I$ that

$$
\frac{\delta_{i} L_{i}}{\delta u_{I i}}=\frac{\delta_{j} L_{j}}{\delta u_{I j}}
$$

It is clear these equations combined are also sufficient for the action to be critical.

\subsection{Multi-time Euler-Lagrange Equations for Two-Dimensional Surfaces}

The two-dimensional case $(d=2)$ covers many known integrable hierarchies, including the potential $\mathrm{KdV}$ hierarchy which we will discuss in detail later on. We consider a Lagrangian two-form $\mathscr{L}=\sum_{i<j} L_{i j} \mathrm{~d} t_{i} \wedge \mathrm{d} t_{j}$ and we will use the notational convention $L_{j i}=-L_{i j}$. 
Theorem 2.7 The multi-time Euler-Lagrange equations for two-dimensional surfaces are

$$
\begin{array}{lr}
\frac{\delta_{i j} L_{i j}}{\delta u_{I}}=0, & \forall I \not \ngtr i, j, \\
\frac{\delta_{i j} L_{i j}}{\delta u_{I j}}=\frac{\delta_{i k} L_{i k}}{\delta u_{I k}} & \forall I \not \ni i, \\
\frac{\delta_{i j} L_{i j}}{\delta u_{I i j}}+\frac{\delta j k}{\delta u_{I j k}}+\frac{\delta_{k i} L_{k i}}{\delta u_{I k i}}=0 & \forall I,
\end{array}
$$

where $i, j$ and $k$ are distinct, and the following notation is used for the variational derivative corresponding to the coordinate directions $i, j$ :

$$
\frac{\delta_{i j} L_{i j}}{\delta u_{I}}:=\sum_{\alpha, \beta \geq 0}(-1)^{\alpha+\beta} \mathrm{D}_{i}^{\alpha} \mathrm{D}_{j}^{\beta} \frac{\partial L_{i j}}{\partial u_{I i^{\alpha} j^{\beta}}} .
$$

Remark 2.8 In the special case that $\mathscr{L}$ only depends on the second jet bundle, this system reduces to the equations stated in [21].

Before proceeding with the proof of Theorem 2.7, we introduce some terminology and prove a lemma. A two-dimensional stepped surface consisting of $q$ flat pieces intersecting at some point $p$ is called a $q$-flower around $p$, the flat pieces are called its petals. If the action is stationary on every $q$-flower, it is stationary on any stepped surface. By Lemma 2.4 the action will then be stationary on any surface. The following Lemma shows that it is sufficient to consider 3 -flowers.

Lemma 2.9 If the action is stationary on every 3-flower, then it is stationary on every $q$-flower for any $q>3$.

Proof Let $F$ be a $q$-flower. Denote its petals corresponding to coordinate directions $\left(t_{i_{1}}, t_{i_{2}}\right),\left(t_{i_{2}}, t_{i_{3}}\right), \ldots,\left(t_{i_{q}}, t_{i_{1}}\right)$ by $S_{12}, S_{23}, \ldots, S_{q 1}$ respectively. Consider the 3 -flower $F_{123}=S_{12} \cup S_{23} \cup S_{31}$, where $S_{31}$ is a petal in the coordinate direction $\left(t_{i_{3}}, t_{i_{1}}\right)$ such that $F_{123}$ is a flower around the same point as F. Similarly, define $F_{134}, \ldots, F_{1 q-1 q}$. Then (for any integrand)

$$
\begin{aligned}
\int_{F_{123}}+\int_{F_{134}}+\ldots+\int_{F_{1 q-1 q}} & \\
& =\int_{S_{12}}+\int_{S_{23}}+\int_{S_{31}}+\int_{S_{13}}+\int_{S_{34}}+\int_{S_{41}}+\ldots+\int_{S_{1 q-1}}+\int_{S_{q-1 q}}+\int_{S_{q 1}} .
\end{aligned}
$$

Here, $S_{21}, S_{32}, \ldots$ are the petals $S_{12}, S_{23}, \ldots$ but with opposite orientation (see Fig. 2). Therefore all terms where the index of $S$ contains 1 cancel, except for the first and last, leaving

$$
\int_{F_{123}}+\ldots+\int_{F_{1 q-1 q}}=\int_{S_{12}}+\int_{S_{23}}+\int_{S_{34}}+\ldots+\int_{S_{q-1 q}}+\int_{S_{q 1}}=\int_{F} .
$$


Fig. 2 Two 3-flowers composed to form a 4-flower. The common petal does not contribute to the integral because it occurs twice with opposite orientation

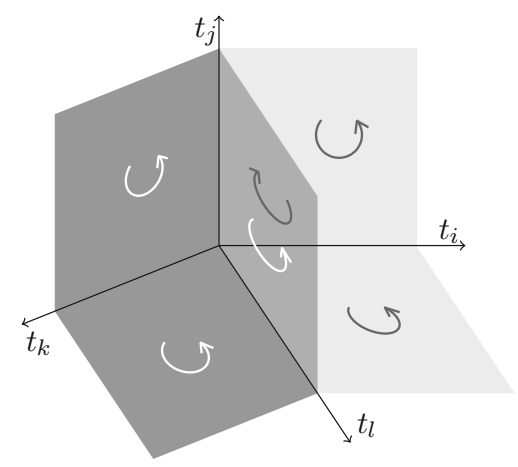

By assumption the action is stationary on every 3-flower, so

$$
\int_{F} \iota_{\operatorname{pr} \mathscr{V}} \delta \mathscr{L}=\int_{F_{123}} \iota_{\operatorname{pr} \mathscr{V}} \delta \mathscr{L}+\ldots+\int_{F_{1 q-1 q}} \iota_{\operatorname{pr} \mathscr{V}} \delta \mathscr{L}=0 .
$$

Proof (of Theorem 2.7) Consider a 3-flower $S=S_{i j} \cup S_{j k} \cup S_{k i}$ around the point $p=S_{i j} \cap S_{j k} \cap S_{k i}$. Denote its interior edges by

$$
\partial S_{i}:=S_{i j} \cap S_{k i}, \quad \partial S_{j}:=S_{j k} \cap S_{i j}, \quad \partial S_{k}:=S_{k i} \cap S_{j k}
$$

On $\partial S_{i}, \partial S_{j}$ and $\partial S_{k}$ we choose the orientations that induce negative orientation on $p$. We consider the case where these orientations correspond to the coordinate directions, as in Fig. 3. The cases where one or more of these orientations are opposite to the corresponding coordinate direction (see Fig. 4) can be treated analogously and yield the same result.

Fig. 3 A 3-flower. Different petals induce the opposite orientation on the common boundary

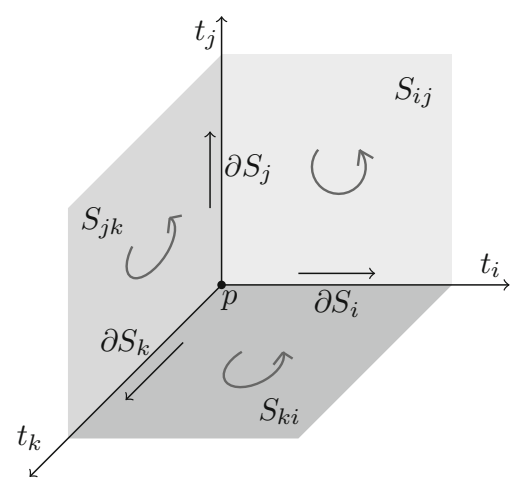



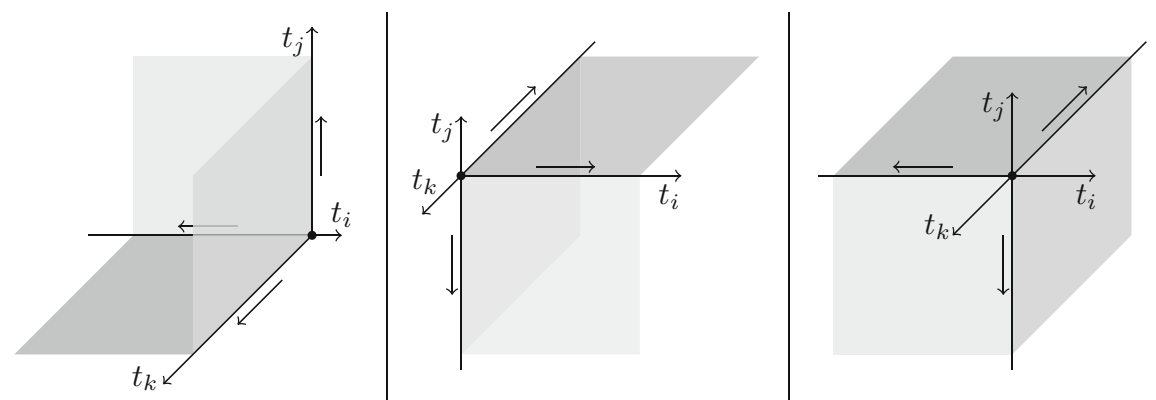

Fig. 4 Three of the other 3-flowers. The orientations of the interior edges do not all correspond to the coordinate direction

We choose the orientation on the petals in such a way that the orientations of $\partial S_{i}$, $\partial S_{j}$ and $\partial S_{k}$ are induced by $S_{i j}, S_{j k}$ and $S_{k i}$ respectively. Then the orientations of $\partial S_{i}, \partial S_{j}$ and $\partial S_{k}$ are the opposite of those induced by $S_{k i}, S_{i j}$ and $S_{j k}$ respectively (see Fig. 3).

We will calculate

$$
\int_{S} \iota_{\mathrm{pr} \mathscr{V}} \delta \mathscr{L}=\int_{S_{i j}} \iota_{\mathrm{pr} \mathscr{V}} \delta \mathscr{L}+\int_{S_{j k}} \iota_{\mathrm{pr} \mathscr{V}} \delta \mathscr{L}+\int_{S_{k i}} \iota_{\mathrm{pr} \mathscr{\mathscr { V }}} \delta \mathscr{L}
$$

and require it to be zero for any variation $\mathscr{V}$ which vanishes on the (outer) boundary of $S$. This will give us the multi-time Euler-Lagrange equations.

For the first term of Eq. (8) we find

$$
\begin{aligned}
\int_{S_{i j}} \iota_{\mathrm{pr} \mathscr{V}} \delta \mathscr{L} & =\int_{S_{i j}} \sum_{I} \frac{\partial L_{i j}}{\partial u_{I}} \delta u_{I}(\mathscr{V}) \mathrm{d} t_{i} \wedge \mathrm{d} t_{j} \\
& =\int_{S_{i j}} \sum_{I \not \supset i, j} \sum_{\lambda, \mu \geq 0} \frac{\partial L_{i j}}{\partial u_{I i^{\lambda} j \mu}} \delta u_{I i^{\lambda} j^{\mu}}(\mathscr{V}) \mathrm{d} t_{i} \wedge \mathrm{d} t_{j} .
\end{aligned}
$$

First we perform integration by parts with respect to $t_{i}$ as many times as possible.

$$
\begin{aligned}
\int_{S_{i j}} \iota_{\mathrm{pr} \mathscr{V}} \delta \mathscr{L}= & \int_{S_{i j}} \sum_{I \not \supset i, j} \sum_{\lambda, \mu \geq 0}(-1)^{\lambda} \mathrm{D}_{i}^{\lambda} \frac{\partial L_{i j}}{\partial u_{I i^{\lambda} j^{\mu}}} \delta u_{I j^{\mu}}(\mathscr{V}) \mathrm{d} t_{i} \wedge \mathrm{d} t_{j} \\
& -\int_{\partial S_{j}} \sum_{I \ngtr \ngtr i, j} \sum_{\lambda, \mu \geq 0} \sum_{\pi=0}^{\lambda-1}(-1)^{\pi} \mathrm{D}_{i}^{\pi} \frac{\partial L_{i j}}{\partial u_{I i^{\lambda} j^{\mu}}} \delta u_{I i^{\lambda-\pi-1} j^{\mu}}(\mathscr{V}) \mathrm{d} t_{j} .
\end{aligned}
$$

Next integrate by parts with respect to $t_{j}$ as many times as possible.

$$
\int_{S_{i j}} \iota_{\mathrm{pr} \mathscr{V}} \delta \mathscr{L}=\int_{S_{i j}} \sum_{I \not \supset i, j} \sum_{\lambda, \mu \geq 0}(-1)^{\lambda+\mu} \mathrm{D}_{i}^{\lambda} \mathrm{D}_{j}^{\mu} \frac{\partial L_{i j}}{\partial u_{I i^{\lambda} j^{\mu}}} \delta u_{I}(\mathscr{V}) \mathrm{d} t_{i} \wedge \mathrm{d} t_{j}
$$




$$
\begin{aligned}
& -\int_{\partial S_{j}} \sum_{I \not i, j} \sum_{\lambda, \mu \geq 0} \sum_{\pi=0}^{\lambda-1}(-1)^{\pi} \mathrm{D}_{i}^{\pi} \frac{\partial L_{i j}}{\partial u_{I i^{\lambda} j^{\mu}}} \delta u_{I i^{\lambda-\pi-1} j^{\mu}}(\mathscr{V}) \mathrm{d} t_{j} \\
& -\int_{\partial S_{i}} \sum_{I \not p i, j} \sum_{\lambda, \mu \geq 0} \sum_{\rho=0}^{\mu-1}(-1)^{\lambda+\rho} \mathrm{D}_{i}^{\lambda} \mathrm{D}_{j}^{\rho} \frac{\partial L_{i j}}{\partial u_{I i^{\lambda} j^{\mu}}} \delta u_{I j^{\mu-\rho-1}}(\mathscr{V}) \mathrm{d} t_{i} .
\end{aligned}
$$

The signs of (10) and (11) are due to the choice of orientations (see Fig. 3). We can rewrite the integral (9) as

$$
\int_{S_{i j}} \sum_{I \not i, j} \frac{\delta_{i j} L_{i j}}{\delta u_{I}} \delta u_{I}(\mathscr{V}) \mathrm{d} t_{i} \wedge \mathrm{d} t_{j} .
$$

The last integral (11) takes a similar form if we replace the index $\mu$ by $\beta=\mu-\rho-1$.

$$
\begin{aligned}
-\int_{\partial S_{i}} \sum_{I \not p i, j} \sum_{\lambda, \mu \geq 0} \sum_{\rho=0}^{\mu-1}(-1)^{\lambda+\rho} \mathrm{D}_{i}^{\lambda} \mathrm{D}_{j}^{\rho} \frac{\partial L_{i j}}{\partial u_{I i^{\lambda} j^{\mu}}} \delta u_{I j^{\mu-\rho-1}}(\mathscr{V}) \mathrm{d} t_{i} \\
=-\int_{\partial S_{i}} \sum_{I \not p i, j} \sum_{\beta, \lambda, \rho \geq 0}(-1)^{\lambda+\rho} \mathrm{D}_{i}^{\lambda} \mathrm{D}_{j}^{\rho} \frac{\partial L_{i j}}{\partial u_{I i^{\lambda} j^{\beta+\rho+1}}} \delta u_{I j^{\beta}}(\mathscr{V}) \mathrm{d} t_{i} \\
=-\int_{\partial S_{i}} \sum_{I \not p i, j} \sum_{\beta \geq 0} \frac{\delta_{i j} L_{i j}}{\delta u_{I j^{\beta+1}}} \delta u_{I j^{\beta}}(\mathscr{V}) \mathrm{d} t_{i} .
\end{aligned}
$$

To write the other boundary integral (10) in this form we first perform integration by parts.

$$
\begin{aligned}
-\int_{\partial S_{j}} \sum_{I \not \supset i, j} \sum_{\lambda, \mu \geq 0} \sum_{\pi=0}^{\lambda-1}(-1)^{\pi} \mathrm{D}_{i}^{\pi} \frac{\partial L_{i j}}{\partial u_{I i^{\lambda} j^{\mu}}} \delta u_{I i^{\lambda-\pi-1} j^{\mu}}(\mathscr{V}) \mathrm{d} t_{j} \\
=-\int_{\partial S_{j}} \sum_{I \not p i, j} \sum_{\lambda, \mu \geq 0} \sum_{\pi=0}^{\lambda-1}(-1)^{\pi+\mu} \mathrm{D}_{i}^{\pi} \mathrm{D}_{j}^{\mu} \frac{\partial L_{i j}}{\partial u_{I i^{\lambda} j^{\mu}}} \delta u_{I i^{\lambda-\pi-1}}(\mathscr{V}) \mathrm{d} t_{j} \\
\quad+\left.\sum_{I \not i, j} \sum_{\lambda, \mu \geq 0} \sum_{\pi=0}^{\lambda-1} \sum_{\rho=0}^{\mu-1}(-1)^{\pi+\rho}\left(\mathrm{D}_{i}^{\pi} \mathrm{D}_{j}^{\rho} \frac{\partial L_{i j}}{\partial u_{I i^{\lambda} j^{\mu}}} \delta u_{I i^{\lambda-\pi-1} j^{\mu-\rho-1}}(\mathscr{V})\right)\right|_{p}
\end{aligned}
$$

Then we replace $\lambda$ by $\alpha=\lambda-\pi-1$ and in the last term $\mu$ by $\beta=\mu-\rho-1$.

$$
\begin{aligned}
& -\int_{\partial S_{j}} \sum_{I \not \supset i, j} \sum_{\lambda, \geq 0} \sum_{\pi=0}^{\lambda-1}(-1)^{\pi} \mathrm{D}_{i}^{\pi} \frac{\partial L_{i j}}{\partial u_{I i^{\lambda} j^{\mu}}} \delta u_{I i^{\lambda-\pi-1} j^{\mu}}(\mathscr{V}) \mathrm{d} t_{j} \\
& =-\int_{\partial S_{j}} \sum_{I \not \supset i, j} \sum_{\alpha, \mu, \pi \geq 0}(-1)^{\pi+\mu} \mathrm{D}_{i}^{\pi} \mathrm{D}_{j}^{\mu} \frac{\partial L_{i j}}{\partial u_{I i^{\alpha+\pi+1} j^{\mu}}} \delta u_{I i^{\alpha}}(\mathscr{V}) \mathrm{d} t_{j}
\end{aligned}
$$




$$
\begin{aligned}
& +\left.\sum_{I \not i, j} \sum_{\alpha, \beta, \pi, \rho \geq 0}\left((-1)^{\pi+\rho} \mathrm{D}_{i}^{\pi} \mathrm{D}_{j}^{\rho} \frac{\partial L_{i j}}{\partial u_{I i^{\alpha+\pi+1} j^{\beta+\rho+1}}} \delta u_{I i^{\alpha} j^{\beta}}(\mathscr{V})\right)\right|_{p} \\
= & -\int_{\partial S_{j}} \sum_{I \not i, j} \sum_{\alpha \geq 0} \frac{\delta_{i j} L_{i j}}{\delta u_{I i^{\alpha+1}}} \delta u_{I i^{\alpha}}(\mathscr{V}) \mathrm{d} t_{j}+\left.\sum_{I \not i, j} \sum_{\alpha, \beta \geq 0}\left(\frac{\delta_{i j} L_{i j}}{\delta u_{I i^{\alpha+1} j^{\beta+1}}} \delta u_{I i^{\alpha} j^{\beta}}(\mathscr{V})\right)\right|_{p}
\end{aligned}
$$

Putting everything together we find

$$
\begin{aligned}
\int_{S_{i j}} \iota_{\operatorname{pr} \mathscr{V}} \delta \mathscr{L}= & \int_{S_{i j}} \sum_{I \not \supset i, j} \frac{\delta_{i j} L_{i j}}{\delta u_{I}} \delta u_{I}(\mathscr{V}) \mathrm{d} t_{i} \wedge \mathrm{d} t_{j}-\int_{\partial S_{i}} \sum_{I \not \supset i} \frac{\delta_{i j} L_{i j}}{\delta u_{I j}} \delta u_{I}(\mathscr{V}) \mathrm{d} t_{i} \\
& -\int_{\partial S_{j}} \sum_{I \not \ngtr j} \frac{\delta_{i j} L_{i j}}{\delta u_{I i}} \delta u_{I}(\mathscr{V}) \mathrm{d} t_{j}+\left.\left(\sum_{I} \frac{\delta_{i j} L_{i j}}{\delta u_{I i j}} \delta u_{I}(\mathscr{V})\right)\right|_{p}
\end{aligned}
$$

Expressions for the integrals over $S_{j k}$ and $S_{k i}$ are found by cyclic permutation of the indices. Finally we obtain

$$
\begin{aligned}
\int_{S} \iota_{\operatorname{pr} \mathscr{V}} \delta \mathscr{L}= & \int_{S_{i j}} \sum_{I \not \ngtr i, j} \frac{\delta_{i j} L_{i j}}{\delta u_{I}} \delta u_{I}(\mathscr{V}) \mathrm{d} t_{i} \wedge \mathrm{d} t_{j} \\
& -\int_{\partial S_{i}}\left(\sum_{I \not \supset i} \frac{\delta_{i j} L_{i j}}{\delta u_{I j}} \delta u_{I}(\mathscr{V})+\sum_{I \not \supset i} \frac{\delta_{k i} L_{k i}}{\delta u_{I k}} \delta u_{I}(\mathscr{V})\right) \mathrm{d} t_{i} \\
& +\left.\sum_{I}\left(\frac{\delta_{i j} L_{i j}}{\delta u_{I i j}} \delta u_{I}(\mathscr{V})\right)\right|_{p}+\text { cyclic permutations in } i, j, k
\end{aligned}
$$

From this we can read off the multi-time Euler-Lagrange equations.

\section{Pluri-Lagrangian Structure of the Sine-Gordon Equation}

We borrow our first example of a pluri-Lagrangian system from [21].

The Sine-Gordon equation $u_{x y}=\sin u$ is the Euler-Lagrange equation for

$$
L=\frac{1}{2} u_{x} u_{y}-\cos u
$$

Consider the vector field $\varphi \frac{\partial}{\partial u}$ with

$$
\varphi=u_{x x x}+\frac{1}{2} u_{x}^{3}
$$


and its prolongation $\mathrm{D}_{\varphi}:=\sum_{I} \varphi_{I} \frac{\partial}{\partial u_{I}}$. It is known that $\mathrm{D}_{\varphi}$ is a variational symmetry for the sine-Gordon equation [18, p. 336]. In particular, we have that

$$
\mathrm{D}_{\varphi} L=\mathrm{D}_{x} N+\mathrm{D}_{y} M
$$

with

$$
\begin{aligned}
& M=\frac{1}{2} \varphi u_{x}-\frac{1}{8} u_{x}^{4}+\frac{1}{2} u_{x x}^{2} \\
& N=\frac{1}{2} \varphi u_{y}-\frac{1}{2} u_{x}^{2} \cos u-u_{x x}\left(u_{x y}-\sin u\right)
\end{aligned}
$$

Now we introduce a new independent variable $z$ corresponding to the "flow" of the generalized vector field $\mathrm{D}_{\varphi}$, i.e. $u_{z}=\varphi$. Consider simultaneous solutions of the Euler-Lagrange equation $\frac{\delta L}{\delta u}=0$ and of the flow $u_{z}=\varphi$ as functions of three independent variables $x, y, z$. Then Eq. (13) expresses the closedness of the two-form

$$
\mathscr{L}=L d x \wedge d y-M d z \wedge d x-N d y \wedge d z
$$

The fact that $\mathrm{d} \mathscr{L}=0$ on solutions is consistent with Proposition 2.2. Hence $\mathscr{L}$ is a reasonable candidate for a Lagrangian two-form.

Theorem 3.1 The multi-time Euler-Lagrange equations for the Lagrangian twoform

$$
\mathscr{L}=L_{12} d x \wedge d y+L_{13} d x \wedge d z+L_{23} d y \wedge d z
$$

with the components

$$
\begin{aligned}
& L_{12}=\frac{1}{2} u_{x} u_{y}-\cos u, \\
& L_{13}=\frac{1}{2} u_{x} u_{z}-\frac{1}{8} u_{x}^{4}+\frac{1}{2} u_{x x}^{2}, \\
& L_{23}=-\frac{1}{2} u_{y} u_{z}+\frac{1}{2} u_{x}^{2} \cos u+u_{x x}\left(u_{x y}-\sin u\right),
\end{aligned}
$$

consist of the sine-Gordon equation

$$
u_{x y}=\sin u
$$

the modified $K d V$ equation

$$
u_{z}=u_{x x x}+\frac{1}{2} u_{x}^{3}
$$

and corollaries thereof. On solutions of either of these equations the two-form $\mathscr{L}$ is closed. 
Proof Let us calculate the multi-time Euler-Lagrange Eqs. (5)-(7) one by one:

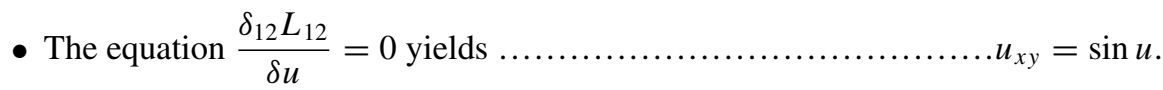

For any $\alpha>0$ the equation $\frac{\delta_{12} L_{12}}{\delta u_{z^{\alpha}}}=0$ yields $0=0$.

- The equation $\frac{\delta_{13} L_{13}}{\delta u}=0$ yields $\ldots \ldots \ldots \ldots \ldots \ldots \ldots \ldots \ldots u_{x z}=\frac{3}{2} u_{x}^{2} u_{x x}+u_{x x x x}$.

For any $\alpha>0$ the equation $\frac{\delta_{13} L_{13}}{\delta u_{y^{\alpha}}}=0$ yields $0=0$.

- The equation $\frac{\delta_{23} L_{23}}{\delta u}=0$ yields $\ldots \ldots \ldots \ldots \ldots \ldots \ldots u_{y z}=\frac{1}{2} u_{x}^{2} \sin u+u_{x x} \cos u$.

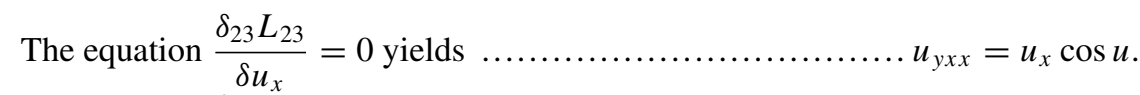

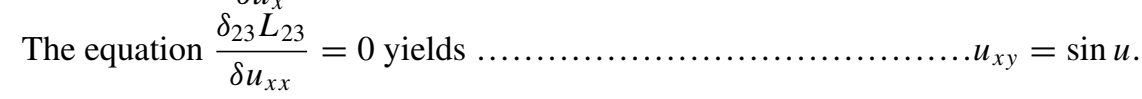

For any $\alpha>2$, the equation $\frac{\delta_{23} L_{23}}{\delta u_{x^{\alpha}}}=0$ yields $0=0$.

- The equation $\frac{\delta_{13} L_{13}}{\delta u_{x}}=\frac{\delta_{23} L_{23}}{\delta u_{y}}$ yields $\ldots \ldots \ldots \ldots \ldots \ldots \ldots \ldots u_{z}=u_{x x x}+\frac{1}{2} u_{x}^{3}$.

The equation $\frac{\delta_{13} L_{13}}{\delta u_{x x}}=\frac{\delta_{23} L_{23}}{\delta u_{x y}}$ yields $u_{x x}=u_{x x}$.

For any other $I$ the equation $\frac{\delta_{13} L_{13}}{\delta u_{I x}}=\frac{\delta_{23} L_{23}}{\delta u_{I y}}$ yields $0=0$.

- The equation $\frac{\delta_{12} L_{12}}{\delta u_{y}}=\frac{\delta_{13} L_{13}}{\delta u_{z}}$ yields $\frac{1}{2} u_{x}=\frac{1}{2} u_{x}$.

For any nonempty $I$, the equation $\frac{\delta_{12} L_{12}}{\delta u_{I y}}=\frac{\delta_{13} L_{13}}{\delta u_{I z}}$ yields $0=0$.

- The equation $\frac{\delta_{12} L_{12}}{\delta u_{x}}=\frac{\delta_{23} L_{32}}{\delta u_{z}}$ yields $\frac{1}{2} u_{y}=\frac{1}{2} u_{y}$.

For any nonempty $I$, the equation $\frac{\delta_{12} L_{12}}{\delta u_{I x}}=\frac{\delta_{23} L_{32}}{\delta u_{I z}}$ yields $0=0$.

- For any $I$ the equation $\frac{\delta_{12} L_{12}}{\delta u_{I x y}}+\frac{\delta_{23} L_{23}}{\delta u_{I y z}}+\frac{\delta_{13} L_{31}}{\delta u_{I z x}}=0$ yields $0=0$.

It remains to notice that all nontrivial equations in this list are corollaries of the equations $u_{x y}=\sin u$ and $u_{z}=u_{x x x}+\frac{1}{2} u_{x}^{3}$, derived by differentiation.

The closedness of $\mathscr{L}$ can be verified by direct calculation:

$\mathrm{D}_{z} L_{12}-\mathrm{D}_{y} L_{13}+\mathrm{D}_{x} L_{23}=\frac{1}{2}\left(u_{y z} u_{x}+u_{x z} u_{y}\right)+u_{z} \sin u$

$$
-\frac{1}{2} u_{y z} u_{x}-\frac{1}{2} u_{z} u_{x y}+\frac{1}{2} u_{x}^{3} u_{x y}-u_{x x} u_{x x y}
$$




$$
\begin{aligned}
& -\frac{1}{2} u_{x z} u_{y}-\frac{1}{2} u_{z} u_{x y}+u_{x} u_{x x} \cos u-\frac{1}{2} u_{x}^{3} \sin u \\
& +u_{x x x}\left(u_{x y}-\sin u\right)+u_{x x}\left(u_{x x y}-u_{x} \cos u\right) \\
= & -\left(u_{z}-\frac{1}{2} u_{x}^{3}-u_{x x x}\right)\left(u_{x y}-\sin u\right) .
\end{aligned}
$$

Remark 3.2 The Sine-Gordon equation and the modified KdV equation are the simplest equations of their respective hierarchies. Furthermore, those hierarchies can be seen as the positive and negative parts of one single hierarchy that is infinite in both directions [14, Sect. 3c and 5k]. It seems likely that this whole hierarchy possesses a pluri-Lagrangian structure.

\section{The KdV Hierarchy}

Our second and the main example of a pluri-Lagrangian system will be the (potential) $\mathrm{KdV}$ hierarchy. This section gives an overview of the relevant known facts about $\mathrm{KdV}$, mainly following Dickey [12, Sect. 3.7]. The next section will present its pluriLagrangian structure.

One way to introduce the Korteweg-de Vries $(K d V)$ hierarchy is to consider a formal power series

$$
R=\sum_{k=0}^{\infty} r_{k} z^{-2 k-1}
$$

with the coefficients $r_{k}=r_{k}[u]$ being polynomials of $u$ and its partial derivatives with respect to $x$, satisfying the equation

$$
R_{x x x}+4 u R_{x}+2 u_{x} R-z^{2} R_{x}=0 .
$$

Multiplying this equation by $R$ and integrating with respect to $x$ we find

$$
R R_{x x}-\frac{1}{2} R_{x}^{2}+2\left(u-\frac{1}{4} z^{2}\right) R^{2}=C(z),
$$

where $C(z)=\sum_{k=0}^{\infty} c_{k} z^{-2 k}$ is a formal power series in $z^{-2}$, with coefficients $c_{k}$ being constants. Different choices of $C(z)$ correspond to different normalizations of the $\mathrm{KdV}$ hierarchy. We take $C(z)=\frac{1}{8}$, i.e. $c_{0}=\frac{1}{8}$ and $c_{k}=0$ for $k>0$. The first few coefficients of the power series $R=r_{0} z^{-1}+r_{1} z^{-3}+r_{2} z^{-5}+\ldots$ are

$$
r_{0}=\frac{1}{2}, \quad r_{1}=u, \quad r_{2}=u_{x x}+3 u^{2}, \quad r_{3}=u_{x x x x}+10 u u_{x x}+5 u_{x}^{2}+10 u^{3} .
$$

The Korteweg-de Vries hierarchy is defined as follows. 
Definition 4.1 - The $K d V$ hierarchy is the family of equations

$$
u_{t_{k}}=\left(r_{k}[u]\right)_{x}
$$

- Write $g_{k}[v]:=r_{k}\left[v_{x}\right]$. The potential $K d V(P K d V)$ hierarchy is the family of equations

$$
v_{t_{k}}=g_{k}[v]
$$

- The differentiated potential $K d V(D P K d V)$ hierarchy is the family of equations

$$
v_{x t_{k}}=\left(g_{k}[v]\right)_{x} .
$$

The right-hand sides of first few PKdV equations are

$$
g_{1}=v_{x}, \quad g_{2}=v_{x x x}+3 v_{x}^{2}, \quad g_{3}=v_{x x x x x}+10 v_{x} v_{x x x}+5 v_{x x}^{2}+10 v_{x}^{3} .
$$

Remark 4.2 The first KdV and PKdV equations, $u_{t_{1}}=u_{x}$, resp. $v_{t_{1}}=v_{x}$, allow us to identify $x$ with $t_{1}$.

Proposition 4.3 The differential polynomials $r_{k}[u]$ satisfy

$$
\frac{\delta r_{k}}{\delta u}=(4 k-2) r_{k-1}
$$

where $\frac{\delta}{\delta u}$ is shorthand notation for $\frac{\delta_{1}}{\delta u}$.

A proof of this statement can be found in [12, 3.7.11-3.7.14].

Corollary 4.4 Set $h_{k}[v]:=\frac{1}{4 k+2} g_{k+1}[v]$, then the differential polynomials $g_{k}$ and $h_{k}$ satisfy

$$
\frac{\delta g_{k}}{\delta v_{x}}=(4 k-2) g_{k-1} \quad \text { and } \quad \frac{\delta h_{k}}{\delta v_{x}}=g_{k} .
$$

Before we proceed, let us formulate a simple Lemma.

Lemma 4.5 For any multi-index I and for any differential polynomial $f[v]$ we have:

$$
\mathrm{D}_{x}\left(\frac{\delta f}{\delta v_{I x}}\right)=\frac{\partial f}{\partial v_{I}}-\frac{\delta f}{\delta v_{I}}
$$

Proof By direct calculation:

$$
\mathrm{D}_{x}\left(\frac{\delta f}{\delta v_{I x}}\right)=\mathrm{D}_{x}\left(\frac{\partial f}{\partial v_{I x}}-\mathrm{D}_{x} \frac{\partial f}{\partial v_{I x^{2}}}+\mathrm{D}_{x}^{2} \frac{\partial f}{\partial v_{I x^{3}}}-\ldots\right)
$$




$$
=\mathrm{D}_{x} \frac{\partial f}{\partial v_{I x}}-\mathrm{D}_{x}^{2} \frac{\partial f}{\partial v_{I x^{2}}}+\mathrm{D}_{x}^{2} \frac{\partial f}{\partial v_{I x^{3}}}-\ldots=\frac{\partial f}{\partial v_{I}}-\frac{\delta f}{\delta v_{I}} .
$$

We can now find Lagrangians for the the DPKdV equations.

Proposition 4.6 The DPKdV equations are Lagrangian, with the Lagrange functions

$$
L_{k}[v]=\frac{1}{2} v_{x} v_{t_{k}}-h_{k}[v]
$$

Proof Since $h_{k}=\frac{1}{4 k+2} g_{k+1}$ does not depend on $v$ directly, it follows from Lemma 4.5 and Corollary 4.4 that

$$
\frac{\delta L_{k}}{\delta v}=-v_{t_{k} x}-\frac{\delta h_{k}}{\delta v}=-v_{t_{k} x}+\mathrm{D}_{x} \frac{\delta h_{k}}{\delta v_{x}}=-v_{t_{k} x}+\left(g_{k}\right)_{x} .
$$

\section{Pluri-Lagrangian Structure of PKdV Hierarchy}

Since the individual $\mathrm{KdV}$ and $\mathrm{PKdV}$ equations are evolutionary (not variational), it seems not very plausible that they could have a pluri-Lagrangian structure. However, it turns out that the PKdV hierarchy as a whole is pluri-Lagrangian. Let us stress that this structure is only visible if one considers several PKdV equations simultaneously and not individually. We consider a finite-dimensional multi-time $\mathbb{R}^{N}$ parametrized by $t_{1}, t_{2}, \ldots, t_{N}$ supporting the first $N$ flows of the PKdV hierarchy. Recall that the first PKdV equation reads $v_{t_{1}}=v_{x}$, which allows us to identify $t_{1}$ with $x$.

The formulation of the main result involves certain differential polynomials introduced in the following statement.

Lemma 5.1 - There exist differential polynomials $b_{i j}[v]$ depending on $v$ and $v_{x^{\alpha}}$, $\alpha>0$, such that

$$
\mathrm{D}_{x}\left(g_{i}\right) g_{j}=\mathrm{D}_{x}\left(b_{i j}\right) .
$$

- These polynomials satisfy

$$
b_{i j}+b_{j i}=g_{i} g_{j}
$$

- The differential polynomials $a_{i j}[v]$ (depending on $v_{x^{\alpha}}$ and $v_{x^{\alpha} t_{j}}, \alpha \geq 0$ ) defined by

$$
a_{i j}:=v_{t_{j}} \frac{\delta_{1} h_{i}}{\delta v_{x}}+v_{x t_{j}} \frac{\delta_{1} h_{i}}{\delta v_{x x}}+v_{x x t_{j}} \frac{\delta_{1} h_{i}}{\delta v_{x x x}}+\ldots
$$


satisfy

$$
\mathrm{D}_{j}\left(h_{i}\right)+\mathrm{D}_{x}\left(g_{i}\right) v_{t_{j}}=\mathrm{D}_{x}\left(a_{i j}\right) .
$$

Proof The existence of polynomials $b_{i j}$ is shown in [12, 3.7.9]. Since

$$
\mathrm{D}_{x}\left(b_{i j}+b_{j i}\right)=\mathrm{D}_{x}\left(g_{i}\right) g_{j}+g_{i} \mathrm{D}_{x}\left(g_{j}\right)=\mathrm{D}_{x}\left(g_{i} g_{j}\right),
$$

and since neither $b_{i j}+b_{j i}$ nor $g_{i} g_{j}$ contain constant terms, Eq. (20) follows. The last claim is a straightforward calculation using Lemma 4.5:

$$
\begin{aligned}
\mathrm{D}_{x}\left(a_{i j}\right)= & \mathrm{D}_{x}\left(v_{t_{j}} \frac{\delta_{1} h_{i}}{\delta v_{x}}+v_{x t_{j}} \frac{\delta_{1} h_{i}}{\delta v_{x x}}+v_{x x t_{j}} \frac{\delta_{1} h_{i}}{\delta v_{x x x}}+\ldots\right) \\
= & v_{x t_{j}} \frac{\delta_{1} h_{i}}{\delta v_{x}}+v_{x x t_{j}} \frac{\delta_{1} h_{i}}{\delta v_{x x}}+v_{x x x t_{j}} \frac{\delta_{1} h_{i}}{\delta v_{x x x}}+\ldots \\
& +v_{t_{j}} \mathrm{D}_{x}\left(\frac{\delta_{1} h_{i}}{\delta v_{x}}\right)+v_{x t_{j}} \mathrm{D}_{x}\left(\frac{\delta_{1} h_{i}}{\delta v_{x x}}\right)+v_{x x t_{j}} \mathrm{D}_{x}\left(\frac{\delta_{1} h_{i}}{\delta v_{x x x}}\right)+\ldots \\
= & v_{x t_{j}} \frac{\delta_{1} h_{i}}{\delta v_{x}}+v_{x x t_{j}} \frac{\delta_{1} h_{i}}{\delta v_{x x}}+v_{x x x t_{j}} \frac{\delta_{1} h_{i}}{\delta v_{x x x}}+\ldots \\
& -v_{t_{j}} \frac{\delta_{1} h_{i}}{\delta v}+v_{t_{j}} \frac{\partial h_{i}}{\partial v}-v_{x t_{j}} \frac{\delta_{1} h_{i}}{\delta v_{x}}+v_{x t_{j}} \frac{\partial h_{i}}{\partial v_{x}}-v_{x x t_{j}} \frac{\delta_{1} h_{i}}{\delta v_{x x}}+v_{x x t_{j}} \frac{\partial h_{i}}{\partial v_{x x}}-\ldots \\
= & \mathrm{D}_{j} h_{i}-v_{t_{j}} \frac{\delta_{1} h_{i}}{\delta v}=\mathrm{D}_{j} h_{i}+\mathrm{D}_{x}\left(g_{i}\right) v_{t_{j}} .
\end{aligned}
$$

Now we are in a position to give a pluri-Lagrangian formulation of the PKdV hierarchy.

Theorem 5.2 The multi-time Euler-Lagrange equations for the Lagrangian twoform $\mathscr{L}=\sum_{i<j} L_{i j} \mathrm{~d} t_{i} \wedge \mathrm{d} t_{j}$, with coefficients given by

$$
L_{1 i}:=L_{i}=\frac{1}{2} v_{x} v_{t_{i}}-h_{i}
$$

and

$$
L_{i j}:=\frac{1}{2}\left(v_{t_{i}} g_{j}-v_{t_{j}} g_{i}\right)+\left(a_{i j}-a_{j i}\right)-\frac{1}{2}\left(b_{i j}-b_{j i}\right) \text { for } j>i>1
$$

are the first $N-1$ nontrivial PKdV equations

$$
v_{t_{2}}=g_{2}, \quad v_{t_{3}}=g_{3}, \quad \ldots \quad v_{t_{N}}=g_{N}
$$

and equations that follow from these by differentiation. 


\subsection{Variational Symmetries and the Pluri-Lagrangian Form}

Before proving Theorem 5.2, let us give an heuristic derivation of expression (24) for $L_{i j}$. The ansatz is that different flows of the PKdV hierarchy should be variational symmetries of each other. (We are grateful to V. Adler who proposed this derivation to us in a private communication.)

Fix two distinct integers $i, j \in\{2,3, \ldots, N\}$. Consider the the $i$ th DPKdV equation, which is nothing but the conventional two-dimensional variational system generated in the $\left(x, t_{i}\right)$-plane by the Lagrange function

$$
L_{1 i}[v]=\frac{1}{2} v_{x} v_{t_{i}}-h_{i}[v]
$$

Consider the evolutionary equation $v_{t_{j}}=g_{j}[v]$, i.e., the $j$ th PKdV equation, and the corresponding generalized vector field

$$
\mathrm{D}_{g_{j}}:=\sum_{I \not \supset j}\left(\mathrm{D}_{I} g_{j}\right) \frac{\partial}{\partial v_{I}} .
$$

We want to show that $\mathrm{D}_{g_{j}}$ is a variational symmetry of $L_{1 i}$. For this end, we look for $L_{i j}$ such that

$$
\mathrm{D}_{g_{j}}\left(L_{1 i}\right)-\mathrm{D}_{i}\left(L_{1 j}^{\left(g_{j}\right)}\right)+\mathrm{D}_{x}\left(L_{i j}\right)=0 .
$$

Here, $L_{1 j}^{\left(g_{j}\right)}$ is the Lagrangian defined by (23) but with $v_{t_{j}}$ replaced by $g_{j}$ :

$$
L_{1 j}^{\left(g_{j}\right)}:=\frac{1}{2} v_{x} g_{j}-h_{j}
$$

We have:

$$
\begin{aligned}
& \mathrm{D}_{i}\left(L_{1 j}^{\left(g_{j}\right)}\right)=\frac{1}{2} v_{t_{i} x} g_{j}+\frac{1}{2} v_{x}\left(g_{j}\right)_{t_{i}}-\mathrm{D}_{i}\left(h_{j}\right), \\
& \mathrm{D}_{g_{j}}\left(L_{1 i}\right)=\frac{1}{2}\left(g_{j}\right)_{x} v_{t_{i}}+\frac{1}{2} v_{x}\left(g_{j}\right)_{t_{i}}-\mathrm{D}_{g_{j}}\left(h_{i}\right) .
\end{aligned}
$$

Upon using (22) and (19), and introducing the polynomial

$$
a_{i j}^{\left(g_{j}\right)}:=g_{j} \frac{\delta_{1} h_{i}}{\delta v_{x}}+\left(g_{j}\right)_{x} \frac{\delta_{1} h_{i}}{\delta v_{x x}}+\left(g_{j}\right)_{x x} \frac{\delta_{1} h_{i}}{\delta v_{x x x}}+\ldots
$$

obtained from $a_{i j}$ through the replacement of $v_{t_{j}}$ by $g_{j}$, we find:

$\mathrm{D}_{i}\left(L_{1 j}^{\left(g_{j}\right)}\right)-\mathrm{D}_{g_{j}}\left(L_{1 i}\right)=\frac{1}{2} v_{t_{i} x} g_{j}-\frac{1}{2}\left(g_{j}\right)_{x} v_{t_{i}}-\mathrm{D}_{i}\left(h_{j}\right)+\mathrm{D}_{g_{j}}\left(h_{i}\right)$ 


$$
\begin{aligned}
& =\frac{1}{2} v_{t_{i} x} g_{j}-\frac{1}{2}\left(g_{j}\right)_{x} v_{t_{i}}-\left(a_{j i}\right)_{x}+\left(g_{j}\right)_{x} v_{t_{i}}+\left(a_{i j}^{\left(g_{j}\right)}\right)_{x}-\left(g_{i}\right)_{x} g_{j} \\
& =\frac{1}{2} v_{t_{i} x} g_{j}+\frac{1}{2}\left(g_{j}\right)_{x} v_{t_{i}}-\left(a_{j i}-a_{i j}^{\left(g_{j}\right)}\right)_{x}-\left(g_{i}\right)_{x} g_{j} \\
& =\frac{1}{2}\left(v_{t_{i}} g_{j}\right)_{x}+\left(a_{i j}^{\left(g_{j}\right)}-a_{j i}\right)_{x}-\left(b_{i j}\right)_{x} .
\end{aligned}
$$

We denote the antiderivative with respect to $x$ of this quantity by

$$
L_{i j}^{(i)}:=\frac{1}{2} v_{t_{i}} g_{j}+\left(a_{i j}^{\left(g_{j}\right)}-a_{j i}\right)-b_{i j}
$$

The analogous calculation with coordinates $x$ and $t_{j}$ yields

$$
\mathrm{D}_{g_{i}}\left(L_{1 j}\right)-\mathrm{D}_{j}\left(L_{1 i}^{\left(g_{i}\right)}\right)=-\frac{1}{2}\left(v_{t_{j}} g_{i}\right)_{x}+\left(a_{i j}-a_{j i}^{\left(g_{i}\right)}\right)_{x}+\left(b_{j i}\right)_{x} .
$$

We denote its antiderivative by

$$
L_{i j}^{(j)}:=-\frac{1}{2} v_{t_{j}} g_{i}+\left(a_{i j}-a_{j i}^{\left(g_{i}\right)}\right)+b_{j i}
$$

Now we look for a differential polynomial $L_{i j}[v]$ depending on the partial derivatives of $v$ with respect to $x, t_{i}$ and $t_{j}$ that reduces to $L_{i j}^{(i)}$ and to $L_{i j}^{(j)}$ after the substitutions $v_{t_{j}}=g_{j}$ and $v_{t_{i}}=g_{i}$, respectively. It turns out that there is a one-parameter family of such functions, given by

$$
L_{i j}=c v_{t_{i}} v_{t_{j}}+\left(a_{i j}-a_{j i}\right)+\left(\frac{1}{2}-c\right) v_{t_{i}} g_{j}-\left(\frac{1}{2}+c\right) v_{t_{j}} g_{i}+\frac{1}{2}\left(b_{j i}-b_{i j}\right)+c g_{i} g_{j}
$$

for $c \in \mathbb{R}$. Checking this is a straightforward calculation using Eq. (20). Our theory does not depend in any essential way on the choice of $L_{i j}$ within this family. For aesthetic reasons we chose $c=0$, which gives us Eq. (24).

Remark 5.3 We could also take $\mathscr{L}$ to be the $c$-linear part of the form we have just obtained, i.e. $\mathscr{L}=\sum_{1<i<j}\left(v_{t_{i}}-g_{i}\right)\left(v_{t_{j}}-g_{j}\right) \mathrm{d} t_{i} \wedge \mathrm{d} t_{j}$. One can think of this as choosing $c=\infty$. Such a two-form $\mathscr{L}$ can be considered for any family of evolutionary equations $v_{t_{i}}=g_{i}[v]$. However, due to the vanishing components $L_{1 i}$, this form $\mathscr{L}$ has no relation to the classical variational formulation of the individual differential equations $v_{x t_{i}}=\left(g_{i}\right)_{x}$.

Eventually, Eq. (25) leads to the following closedness property.

Proposition 5.4 The two-form $\mathscr{L}=\sum_{i<j} L_{i j} \mathrm{~d} t_{i} \wedge \mathrm{d} t_{j}$, with coefficients given by (23) and (24), is closed as soon as $v$ solves all but one of the PKdV equations $v_{t_{2}}=g_{2}, \ldots, v_{t_{N}}=g_{N}$. 
Proof We use the notation

$$
\mathrm{d} \mathscr{L}=\sum_{i<j<k} M_{i j k} \mathrm{~d} t_{i} \wedge \mathrm{d} t_{j} \wedge \mathrm{d} t_{k}, \quad M_{i j k}=\mathrm{D}_{k} L_{i j}-\mathrm{D}_{j} L_{i k}+\mathrm{D}_{i} L_{j k}
$$

We start by showing that $M_{1 j k}=\mathrm{D}_{k} L_{1 j}-\mathrm{D}_{j} L_{1 k}+\mathrm{D}_{x} L_{j k}$ vanishes as soon as either $v_{t_{j}}=g_{j}$ or $v_{t_{k}}=g_{k}$ is satisfied. Indeed, we have:

$$
\begin{aligned}
M_{1 j k}= & \mathrm{D}_{k} L_{1 j}-\mathrm{D}_{j} L_{1 k}+\mathrm{D}_{x} L_{j k} \\
= & \frac{1}{2} v_{t_{j} t_{k}} v_{x}+\frac{1}{2} v_{t_{j}} v_{x t_{k}}-\mathrm{D}_{k} h_{j}-\frac{1}{2} v_{t_{j} t_{k}} v_{x}-\frac{1}{2} v_{t_{k}} v_{x t_{j}}+\mathrm{D}_{j} h_{k} \\
& \quad+\frac{1}{2}\left(v_{x t_{j}} g_{k}+v_{t_{j}} \mathrm{D}_{x} g_{k}-v_{x t_{k}} g_{j}-v_{t_{k}} \mathrm{D}_{x} g_{j}\right) \\
& \quad+\mathrm{D}_{k} h_{j}+v_{t_{k}} \mathrm{D}_{x} g_{j}-\mathrm{D}_{j} h_{k}-v_{t_{j}} \mathrm{D}_{x} g_{k}-\frac{1}{2}\left(g_{k} \mathrm{D}_{x} g_{j}-g_{j} \mathrm{D}_{x} g_{k}\right) \\
= & \frac{1}{2}\left(v_{t_{j}} v_{x t_{k}}-v_{t_{k}} v_{x t_{j}}+v_{x t_{j}} g_{k}-v_{t_{j}} \mathrm{D}_{x} g_{k}\right. \\
& \left.\quad-v_{x t_{k}} g_{j}+v_{t_{k}} \mathrm{D}_{x} g_{j}-g_{k} \mathrm{D}_{x} g_{j}+g_{j} \mathrm{D}_{x} g_{k}\right) \\
= & \frac{1}{2}\left(v_{t_{j}}-g_{j}\right) \mathrm{D}_{x}\left(v_{t_{k}}-g_{k}\right)-\frac{1}{2}\left(v_{t_{k}}-g_{k}\right) \mathrm{D}_{x}\left(v_{t_{j}}-g_{j}\right)
\end{aligned}
$$

For the case $i, j, k>1$, we assume without loss of generality that $v_{t_{i}}=g_{i}$ and $v_{t_{j}}=$ $g_{j}$ are satisfied. We do not assume that $v_{t_{k}}=g_{k}$ holds, and correspondingly we do not make any identification involving $v_{t_{k}}, v_{x t_{k}}, \ldots$ Using Eq. (27), we find:

$$
\begin{aligned}
\mathrm{D}_{x} M_{i j k} & =\mathrm{D}_{x}\left(\mathrm{D}_{k} L_{i j}-\mathrm{D}_{j} L_{i k}+\mathrm{D}_{i} L_{j k}\right) \\
& =\mathrm{D}_{k}\left(\mathrm{D}_{i} L_{1 j}-\mathrm{D}_{j} L_{1 i}\right)-\mathrm{D}_{j}\left(\mathrm{D}_{i} L_{1 k}-\mathrm{D}_{k} L_{1 i}\right)+\mathrm{D}_{i}\left(\mathrm{D}_{j} L_{1 k}-\mathrm{D}_{k} L_{1 j}\right) \\
& =0 .
\end{aligned}
$$

Since these polynomials do not contain constant terms, it follows that

$$
\mathrm{D}_{k} L_{i j}-\mathrm{D}_{j} L_{i k}+\mathrm{D}_{i} L_{j k}=0 \text {. }
$$

Remark 5.5 Assuming that the statement of Theorem 5.2 holds true, one can easily prove a somewhat weaker claim than Proposition 5.4, namely that the two-form $\mathscr{L}$ is closed on simultaneous solutions of all the PKdV equations. Indeed, by Proposition 2.2, $\mathrm{d} \mathscr{L}$ is constant on solutions of the multi-time Euler-Lagrange equations $v_{t_{i}}=g_{i}$. Vanishing of this constant follows from the fact that $\mathrm{d} \mathscr{L}=0$ on the trivial solution $v \equiv 0$. 


\subsection{The Multi-time Euler-Lagrange Equations}

Proof (of Theorem 5.2) We check all multi-time Euler-Lagrange Eqs. (5)-(7) individually. If $N>3$, we fix $k>j>i>1$. If $N=3$, we take $j=3, i=2$, and in the following ignore all equations containing $k$. We use the convention $L_{j i}=-L_{i j}$, etc.

\section{Equations (7)}

- The equations

$$
\frac{\delta_{1 i} L_{1 i}}{\delta v_{I x t_{i}}}+\frac{\delta_{i j} L_{i j}}{\delta v_{I t_{i} t_{j}}}+\frac{\delta_{1 j} L_{j 1}}{\delta v_{I t_{j} x}}=0
$$

and

$$
\frac{\delta_{i j} L_{i j}}{\delta v_{I t_{i} t_{j}}}+\frac{\delta_{j k} L_{j k}}{\delta v_{I t_{j} t_{k}}}+\frac{\delta_{k i} L_{k i}}{\delta v_{I t_{k} t_{i}}}=0
$$

are trivial because all terms vanish.

\section{Equations (6)}

- The equation

$$
\frac{\delta_{1 i} L_{1 i}}{\delta v_{x}}=\frac{\delta_{i j} L_{j i}}{\delta v_{t_{j}}}
$$

yields

$$
\begin{aligned}
\frac{1}{2} v_{t_{i}}-\frac{\delta_{1 i} h_{i}}{\delta v_{x}} & =\frac{1}{2} g_{i}-\frac{\delta_{i j} a_{i j}}{\delta v_{t_{j}}} \\
& =\frac{1}{2} g_{i}-\frac{\delta_{i j}}{\delta v_{t_{j}}}\left(v_{t_{j}} \frac{\delta_{1} h_{i}}{\delta v_{x}}+v_{t_{j} x} \frac{\delta_{1} h_{i}}{\delta v_{x x}}+v_{t_{j} x x} \frac{\delta_{1} h_{i}}{\delta v_{x x x}}+\ldots\right) \\
& =\frac{1}{2} g_{i}-\frac{\delta_{1} h_{i}}{\delta v_{x}} .
\end{aligned}
$$

This simplifies to the PKdV equation

$$
v_{t_{i}}=g_{i}
$$

- For $\alpha>0$, the equation

$$
\frac{\delta_{1 i} L_{1 i}}{\delta v_{x^{\alpha+1}}}=\frac{\delta_{i j} L_{j i}}{\delta v_{t_{j} x^{\alpha}}}
$$

yields

$$
-\frac{\delta_{1 i} h_{i}}{\delta v_{x^{\alpha+1}}}=-\frac{\delta_{i j}}{\delta v_{t_{j} x^{\alpha}}}\left(v_{t_{j}} \frac{\delta_{1} h_{i}}{\delta v_{x}}+v_{t_{j} x} \frac{\delta_{1} h_{i}}{\delta v_{x x}}+v_{t_{j} x x} \frac{\delta_{1} h_{i}}{\delta v_{x x x}}+\ldots\right)
$$




$$
=-\frac{\delta_{1} h_{i}}{\delta v_{x^{\alpha+1}}}
$$

which is trivial.

- Similarly, the equation

$$
\frac{\delta_{1 j} L_{1 j}}{\delta v_{x}}=\frac{\delta_{i j} L_{i j}}{\delta v_{t_{i}}}
$$

yields PKdV equation

$$
v_{t_{j}}=g_{j},
$$

and for $\alpha>0$, the equation

$$
\frac{\delta_{1 j} L_{1 j}}{\delta v_{x^{\alpha+1}}}=\frac{\delta_{i j} L_{i j}}{\delta v_{t_{i} x^{\alpha}}}
$$

is trivial.

- All equations of the form

$$
\frac{\delta_{1 i} L_{1 i}}{\delta v_{x I}}=\frac{\delta_{i j} L_{j i}}{\delta v_{t_{j} I}} \quad\left(t_{i} \notin I\right) \quad \text { and } \quad \frac{\delta_{1 j} L_{1 j}}{\delta v_{x I}}=\frac{\delta_{i j} L_{i j}}{\delta v_{t_{i} I}} \quad\left(t_{j} \notin I\right)
$$

where $I$ contains any $t_{l}(l>1)$ are trivial because each term is zero.

- The equations

$$
\frac{\delta_{1 i} L_{1 i}}{\delta v_{I t_{i}}}=\frac{\delta_{1 j} L_{1 j}}{\delta v_{I t_{j}}} \quad(x \notin I)
$$

and

$$
\frac{\delta_{i j} L_{i j}}{\delta v_{I t_{j}}}=\frac{\delta_{i k} L_{i k}}{\delta v_{I t_{k}}} \quad\left(t_{i} \notin I\right)
$$

are easily seen to be trivial as well.

\section{Equations (5)}

- By construction, the equations $\frac{\delta_{1 i} L_{1 i}}{\delta v}=0$ for $i>1$ are the equations

$$
v_{x t_{i}}=\mathrm{D}_{x} g_{i} .
$$

For $I$ containing any $t_{l}, l>1, l \neq i$, the equations $\frac{\delta_{1 i} L_{1 i}}{\delta v_{t_{I}}}=0$ are trivial.

- The last family of equations we discuss as a lemma because its calculation is far from trivial.

Lemma 5.6 The equations $\frac{\delta_{i j} L_{i j}}{\delta v_{x^{\alpha}}}=0$ are corollaries of the PKdV equations. 
Proof (of Lemma 5.6) From Eq. (24) we see that the variational derivative of $L_{i j}$ contains only three nonzero terms,

$$
\frac{\delta_{i j} L_{i j}}{\delta v_{x^{\alpha}}}=\frac{\partial L_{i j}}{\partial v_{x^{\alpha}}}-\mathrm{D}_{i}\left(\frac{\partial L_{i j}}{\partial v_{x^{\alpha} t_{i}}}\right)-\mathrm{D}_{j}\left(\frac{\partial L_{i j}}{\partial v_{x^{\alpha} t_{j}}}\right) .
$$

In particular, the equation $\frac{\delta_{i j} L_{i j}}{\delta v}=0$ yields $\mathrm{D}_{i} g_{j}-\mathrm{D}_{j} g_{i}=0$, that is, the compatibility condition of the flows $v_{t_{i}}=g_{i}$ and $v_{t_{j}}=g_{j}$. To determine the first term on the right hand side of Eq. (31) for an arbitrary $\alpha>0$, we use an indirect method. Assume that the dimension of multi-time $N$ is at least 4 and fix $k>1$ distinct from $i$ and $j$. Let $v$ be a solution of all PKdV equations except $v_{t_{k}}=g_{k}$. By Proposition 5.4 we have

$$
\sum_{I} \frac{\partial L_{i j}}{\partial v_{I}} v_{I t_{k}}=\mathrm{D}_{k} L_{i j}=\mathrm{D}_{j} L_{i k}-\mathrm{D}_{i} L_{j k}
$$

Since $\frac{\partial L_{i j}}{\partial v_{I}}$ does not contain any derivatives with respect to $t_{k}$, we can determine $\frac{\partial L_{i j}}{\partial v_{x^{\alpha}}}$ by looking at the terms in the right hand side of Eq. (32) containing $v_{x^{\alpha}} t_{k}$. These are

$$
\begin{gathered}
\mathrm{D}_{j}\left(-\frac{1}{2} g_{i} v_{t_{k}}+v_{t_{k}} \frac{\delta_{1} h_{i}}{\delta v_{x}}+v_{x t_{k}} \frac{\delta_{1} h_{i}}{\delta v_{x x}}+\ldots\right) \\
-\mathrm{D}_{i}\left(-\frac{1}{2} g_{j} v_{t_{k}}+v_{t_{k}} \frac{\delta_{1} h_{j}}{\delta v_{x}}+v_{x t_{k}} \frac{\delta_{1} h_{j}}{\delta v_{x x}}+\ldots\right) .
\end{gathered}
$$

Now we expand the brackets. By again throwing out all terms that do not contain any $v_{x^{\alpha} t_{k}}$, and those that cancel modulo $v_{t_{i}}=g_{i}$ or $v_{t_{j}}=g_{j}$, we get

$$
\begin{aligned}
& -v_{t_{k}} \mathrm{D}_{j}\left(\frac{\delta_{1} h_{i}}{\delta v_{x}}\right)+v_{x t_{k}} \mathrm{D}_{j}\left(\frac{\delta_{1} h_{i}}{\delta v_{x x}}\right)+v_{x x t_{k}} \mathrm{D}_{j}\left(\frac{\delta_{1} h_{i}}{\delta v_{x x x}}\right)+\ldots \\
& +v_{t_{k}} \mathrm{D}_{i}\left(\frac{\delta_{1} h_{j}}{\delta v_{x}}\right)-v_{x t_{k}} \mathrm{D}_{i}\left(\frac{\delta_{1} h_{j}}{\delta v_{x x}}\right)-v_{x x t_{k}} \mathrm{D}_{i}\left(\frac{\delta_{1} h_{j}}{\delta v_{x x x}}\right)-\ldots
\end{aligned}
$$

Comparing this to Eq. (32), we find that

$$
\frac{\partial L_{i j}}{\partial v_{x^{\alpha}}}=-\mathrm{D}_{i}\left(\frac{\delta_{1} h_{j}}{\delta v_{x^{\alpha+1}}}\right)+\mathrm{D}_{j}\left(\frac{\delta_{1} h_{i}}{\delta v_{x^{\alpha+1}}}\right) .
$$

On the other hand we have

$$
-\mathrm{D}_{i}\left(\frac{\partial L_{i j}}{\partial v_{x^{\alpha} t_{i}}}\right)-\mathrm{D}_{j}\left(\frac{\partial L_{i j}}{\partial v_{x^{\alpha} t_{j}}}\right)=\mathrm{D}_{i}\left(\frac{\delta_{1} h_{j}}{\delta v_{x^{\alpha+1}}}\right)-\mathrm{D}_{j}\left(\frac{\delta_{1} h_{i}}{\delta v_{x^{\alpha+1}}}\right),
$$


so Equation (31) implies that $\frac{\delta_{i j} L_{i j}}{\delta v_{x^{\alpha}}}=0$ for any $\alpha$.

Since $\frac{\delta_{23} L_{23}}{\delta v_{x^{\alpha}}}=0$ does not depend on the dimension $N \geqslant 3$, the result for $N \geqslant 4$ implies the claim for $N=3$.

This concludes the proof of Theorem 5.2.

\section{Relation to Hamiltonian Formalism}

In this last section, we briefly discuss the connection between the closedness of $\mathscr{L}$ and the involutivity of the corresponding Hamiltonians.

In Proposition 2.2 we saw that $\mathrm{d} \mathscr{L}$ is constant on solutions. For the onedimensional case $(d=1)$ with $\mathscr{L}$ depending on the first jet bundle only, it has been shown in [20] that this is equivalent to the commutativity of the corresponding Hamiltonian flows. If the constant is zero then the Hamiltonians are in involution. Now we will prove a similar result for the two-dimensional case.

We will use a Poisson bracket on formal integrals, i.e. equivalence classes of functions modulo $x$-derivatives [12, Chap. 1-2]. In this section, the integral sign $\int$ will always denote an equivalence class, not an integration operator. The Poisson bracket due to Gardner-Zakharov-Faddeev is defined by

$$
\left\{\int F, \int G\right\}=\int\left(\mathrm{D}_{x} \frac{\delta_{1} F}{\delta u}\right) \frac{\delta_{1} G}{\delta u} .
$$

Using integration by parts, we see that this bracket is anti-symmetric. Less obvious is the fact that it satisfies the Jacobi identity [18, Chap. 7]. As we did when studying the KdV hierarchy, we introduce a potential $v$ that satisfies $v_{x}=u$, and we identify the space-coordinate $x$ with the first coordinate $t_{1}$ of multi-time. We can now re-write the Poisson bracket as

$$
\left\{\int F, \int G\right\}=\int\left(\mathrm{D}_{x} \frac{\delta_{1} F}{\delta v_{x}}\right) \frac{\delta_{1} G}{\delta v_{x}}=-\int \frac{\delta_{1} F}{\delta v} \frac{\delta_{1} G}{\delta v_{x}},
$$

for functions $F$ and $G$ that depend on the $x$-derivatives of $v$ but not on $v$ itself.

Assume that the coefficients $L_{1 j}$ of the Lagrangian two-from $\mathscr{L}$ are given by

$$
L_{1 j}=\frac{1}{2} v_{x} v_{t_{j}}-h_{j}
$$

where $h_{j}$ is a differential polynomial in $v_{x}, v_{x x}, \ldots$. This is the case for the PKdV hierarchy. The $L_{1 j}$ are Lagrangians of the equations

$$
v_{x t_{j}}=\mathrm{D}_{x} g_{j} \quad \text { or } \quad u_{t_{j}}=\mathrm{D}_{x} g_{j}
$$


where $g_{j}:=\frac{\delta_{1} h_{j}}{\delta v_{x}}$, hence $\frac{\delta_{1} h_{j}}{\delta v}=-\mathrm{D}_{x} g_{j}$. It turns out that the formal integral $\int h_{j}$ is the Hamilton functional for the equation $u_{t_{j}}=\mathrm{D}_{x} g_{j}$ with respect to the Poisson bracket (33). Formally:

$$
\left\{\int h_{j}, u(y)\right\}=\left\{\int h_{j}, \int u \delta(\cdot-y)\right\}=-\int \frac{\delta_{1} h_{j}}{\delta v} \delta(x-y)=\mathrm{D}_{x} g_{j}(y),
$$

where $\delta$ denotes the Dirac delta.

Theorem 6.1 If $\mathrm{d} \mathscr{L}=0$ on solutions, then the Hamiltonians are in involution,

$$
\left\{\int h_{i}, \int h_{j}\right\}=0
$$

Proof Recall notation (26). We have

$$
\begin{aligned}
\int M_{1 j k} & =\int\left(\mathrm{D}_{x} L_{j k}-\mathrm{D}_{j} L_{1 k}+\mathrm{D}_{k} L_{1 j}\right) \\
& =\int\left(-\mathrm{D}_{j} L_{1 k}+\mathrm{D}_{k} L_{1 j}\right) \\
& =\int\left(-\frac{1}{2} v_{x t_{j}} v_{t_{k}}-\frac{1}{2} v_{x} v_{t_{k} t_{j}}+\mathrm{D}_{j} h_{k}+\frac{1}{2} v_{x t_{k}} v_{t_{j}}+\frac{1}{2} v_{x} v_{t_{j} t_{k}}-\mathrm{D}_{k} L_{1 j}\right) \\
& =\int\left(\frac{1}{2}\left(v_{x t_{k}} v_{t_{j}}-v_{x t_{j}} v_{t_{k}}\right)-\mathrm{D}_{k} L_{1 j}+\mathrm{D}_{j} h_{k}\right)
\end{aligned}
$$

Using Eq. (21) (which, as opposed to Eq. (19), is independent of the form of $h_{i}$ and $g_{i}$ ), the evolution equations $v_{t_{j}}=g_{j}$, and integration by parts, we find that

$$
\begin{aligned}
\int M_{1 j k} & =\int\left(\frac{1}{2}\left(v_{x t_{k}} v_{t_{j}}-v_{x t_{j}} v_{t_{k}}\right)-\mathrm{D}_{x} a_{j k}+v_{t_{k}} \mathrm{D}_{x} g_{j}+\mathrm{D}_{x} a_{k j}-v_{t_{j}} D_{x} g_{k}\right) \\
& =\int\left(-\frac{1}{2}\left(g_{j} \mathrm{D}_{x} g_{k}-g_{k} \mathrm{D}_{x} g_{j}\right)-\mathrm{D}_{x} a_{j k}+\mathrm{D}_{x} a_{k j}\right) \\
& =\int g_{k} \mathrm{D}_{x} g_{j} \\
& =-\int \frac{\delta_{1} h_{j}}{\delta v} \frac{\delta_{1} h_{k}}{\delta v_{x}} \\
& =\left\{\int h_{j}, \int h_{k}\right\} .
\end{aligned}
$$

Hence if $\mathrm{d} \mathscr{L}=0$ on solutions of the evolution equations $v_{t_{j}}=g_{j}$, then the Hamilton functionals are in involution. 


\section{Conclusion}

We have formulated the pluri-Lagrangian theory of integrable hierarchies, and propose it as a definition of integrability. The motivation for this definition comes from the discrete case $[10,13,20]$ and the fact that we have established a relation with the Hamiltonian side of the theory. For the Hamiltonians to be in involution, we need the additional fact that the Lagrangian two-form is closed. However, we believe that the essential part of the theory is inherently contained in the pluri-Lagrangian formalism.

Since the KdV hierarchy is one of the most important examples of an integrable hierarchy, our construction of a pluri-Lagrangian structure for the PKdV hierarchy is an additional indication that the existence of a pluri-Lagrangian structure is a reasonable definition of integrability.

It is remarkable that multi-time Euler-Lagrange equations are capable of producing evolutionary equations. This is a striking difference from the discrete case, where the evolution equations (quad equations) imply the multi-time Euler-Lagrange equations (corner equations), but are themselves not variational [10].

Acknowledgments This research is supported by the Berlin Mathematical School and the DFG Collaborative Research Center TRR 109 "Discretization in Geometry and Dynamics".

\section{A. A very short introduction to the variational bicomplex}

Here we introduce the variational bicomplex and derive the basic results that we use in the text. We follow Dickey, who provides a more complete discussion in [12, Chap. 19]. Another good source on a (subtly different) variational bicomplex is Anderson's unfinished manuscript [2]. For ease of notation we restrict to real fields $u: \mathbb{R}^{N} \rightarrow \mathbb{R}$, rather than vector-valued fields.

The space of $(p, q)$-forms $\mathscr{A}^{(p, q)}$ consists of all formal sums

$$
\omega^{p, q}=\sum f \delta u_{I_{1}} \wedge \ldots \wedge \delta u_{I_{p}} \wedge \mathrm{d} t_{j_{1}} \wedge \ldots \wedge \mathrm{d} t_{j_{q}}
$$

where $f$ is a polynomial in $u$ and partial derivatives of $u$ of arbitrary order with respect to any coordinates. The vertical one-forms $\delta u_{I}$ are dual to the vector fields $\frac{\partial}{\partial u_{I}}$. The action of the derivative $\mathrm{D}_{i}$ on $\omega^{p, q}$ is

$$
\begin{aligned}
\mathrm{D}_{i} \omega^{p, q}=\sum\left(\mathrm{D}_{i} f\right) \delta u_{I_{1}} \wedge \ldots \wedge \delta u_{I_{p}} \wedge \mathrm{d} t_{j_{1}} \wedge \ldots \wedge \mathrm{d} t_{j_{q}} \\
\quad+f \delta u_{I_{1} i} \wedge \ldots \wedge \delta u_{I_{p}} \wedge \mathrm{d} t_{j_{1}} \wedge \ldots \wedge \mathrm{d} t_{j_{q}} \\
+\ldots+f \delta u_{I_{1}} \wedge \ldots \wedge \delta u_{I_{p} i} \wedge \mathrm{d} t_{j_{1}} \wedge \ldots \wedge \mathrm{d} t_{j_{q}}
\end{aligned}
$$

The integral of $\omega^{p, q}$ over an $q$-dimensional manifold is the $(p, 0)$-form defined by 


$$
\int \omega^{p, q}=\sum\left(\int f \mathrm{~d} t_{j_{1}} \wedge \ldots \wedge \mathrm{d} t_{j_{q}}\right) \delta u_{I_{1}} \wedge \ldots \wedge \delta u_{I_{p}} .
$$

We call $(0, q)$-forms horizontal and $(p, 0)$-forms vertical. The horizontal exterior derivative $\mathrm{d}: \mathscr{A}^{(p, q)} \rightarrow \mathscr{A}^{(p, q+1)}$ and the vertical exterior derivative $\delta: \mathscr{A}^{(p, q)} \rightarrow$ $\mathscr{A}^{(p+1, q)}$ are defined by the anti-derivation property

(a) $\mathrm{d}\left(\omega_{1}^{p_{1}, q_{1}} \wedge \omega_{2}^{p_{2}, q_{2}}\right)=\mathrm{d} \omega_{1}^{p_{1}, q_{1}} \wedge \omega_{2}^{p_{2}, q_{2}}+(-1)^{p_{1}+q_{1}} \omega_{1}^{p_{1}, q_{1}} \wedge \mathrm{d} \omega_{2}^{p_{2}, q_{2}}$,

$$
\delta\left(\omega_{1}^{p_{1}, q_{1}} \wedge \omega_{2}^{p_{2}, q_{2}}\right)=\delta \omega_{1}^{p_{1}, q_{1}} \wedge \omega_{2}^{p_{2}, q_{2}}+(-1)^{p_{1}+q_{1}} \omega_{1}^{p_{1}, q_{1}} \wedge \delta \omega_{2}^{p_{2}, q_{2}},
$$

and by the way they act on $(0,0)-,(1,0)-$, and $(0,1)$-forms:

$$
\begin{aligned}
& \text { (b) } \mathrm{d} f=\sum_{j} \mathrm{D}_{j} f \mathrm{~d} t_{j}=\sum_{j}\left(\frac{\partial f}{\partial t_{j}}+\sum_{I} \frac{\partial f}{\partial u_{I}} u_{I j}\right) \mathrm{d} t_{j}, \quad \delta f=\sum_{I} \frac{\partial f}{\partial u_{I}} \delta u_{I}, \\
& \text { (c) } \mathrm{d}\left(\delta u_{I}\right)=-\sum_{j} \delta u_{I j} \wedge \mathrm{d} t_{j}, \quad \delta\left(\delta u_{I}\right)=0, \\
& \text { (d) } \mathrm{d}\left(\mathrm{d} t_{j}\right)=0, \quad \delta\left(\mathrm{d} t_{j}\right)=0, \quad \delta\left(\mathrm{d} u_{I}\right)=-\mathrm{d}\left(\delta u_{I}\right)=\sum_{j} \delta u_{I j} \wedge \mathrm{d} t_{j} .
\end{aligned}
$$

Properties (a)-(d) determine the action of $\mathrm{d}$ and $\delta$ on any form. The corresponding mapping diagram is known as the variational bicomplex.

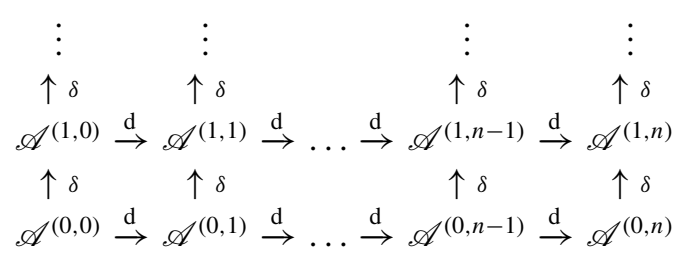

The following claims follow immediately from the definitions.

Proposition A.1 We have $\mathrm{d}^{2}=\delta^{2}=0$ and $\mathrm{d} \delta+\delta \mathrm{d}=0$.

Remark A.2 This implies that $\mathrm{d}+\delta: \mathscr{A}^{k} \rightarrow \mathscr{A}^{k+1}$, where $\mathscr{A}^{k}:=\bigcup_{i=0}^{k} \mathscr{A}^{(i, k-i)}$, is an exterior derivative as well.

Proposition A.3 We have $\mathrm{D}_{i} \delta=\delta \mathrm{D}_{i}$.

Proposition A.4 For a differential polynomial $h$, define the corresponding vertical generalized vector field by $\partial_{h}:=\sum_{I} h_{I} \frac{\partial}{\partial u_{I}}$. We have $\mathrm{d} \iota_{\partial_{h}}+\iota_{\partial_{h}} \mathrm{~d}=0$.

Proof It suffices to show this for $(0,0)$-forms (polynomials $f$ in $u$ and partial derivatives of $u$ ), for $(0,1)$-forms $\mathrm{d} t_{j}$, and for $(1,0)$-forms $\delta u_{I}$. For $(0,0)$-forms, both terms of the claimed identity are zero:

$$
\mathrm{d}\left(\iota_{\partial_{h}} f\right)=0, \quad \iota_{\partial_{h}}(\mathrm{~d} f)=\iota_{\partial_{h}}\left(\sum_{j} \mathrm{D}_{j} f \mathrm{~d} t_{j}\right)=0 .
$$


Likewise for (0,1)-forms:

$$
\mathrm{d}\left(\iota_{\partial_{h}} \mathrm{~d} t_{j}\right)=0, \quad \iota_{\partial_{h}}\left(\mathrm{dd} t_{j}\right)=0
$$

For $(1,0)$-forms we find:

$$
\iota_{\partial_{h}}\left(\mathrm{~d} \delta u_{I}\right)=\iota_{\partial_{h}}\left(-\sum_{j} \delta u_{I j} \wedge \mathrm{d} t_{j}\right)=-\sum_{j} h_{I j} \mathrm{~d} t_{j}=-\mathrm{d} h_{I}=-\mathrm{d}\left(\iota_{\partial_{h}} \delta u_{I}\right)
$$

\section{B. Proof of Lemma 2.4}

Assume that the action is stationary on all $d$-dimensional stepped surfaces in $\mathbb{R}^{N}$. Let $S$ be a smooth $d$-dimensional surface in $\mathbb{R}^{N}$. Partition the space $\mathbb{R}^{N}$ into hypercubes $C_{i}$ of edge length $\varepsilon$. We can choose this partitioning in such a way that the surface $S$ does not contain the center of any of the hypercubes. Denote $S_{i}^{N}:=S \cap C_{i}$.

We give each hypercube its own coordinate system $[-1,1]^{N} \rightarrow C_{i}$ and identify the hypercube with its coordinates. In each punctured hypercube $[-1,1]^{N} \backslash\{0\}$ we define a family of balloon maps

$$
\mathscr{B}_{\alpha}^{N}:[-1,1]^{N} \backslash\{0\} \rightarrow[-1,1]^{N} \backslash\{0\}: x \mapsto \begin{cases}\frac{\alpha x}{\|x\|_{\max }} & \text { if }\|x\|_{\max }<\alpha \\ x & \text { if }\|x\|_{\max } \geqslant \alpha\end{cases}
$$

for $\alpha \in[0,1]$. Here, $\|x\|_{\max }:=\max \left(\left|x_{1}\right|, \ldots\left|x_{N}\right|\right)$ denotes the maximum norm with respect to the local coordinates. The idea is that from the center of each hypercube, we inflate a square balloon which pushes the curve away from the center, until it lies on the boundary of the hypercube.

Indeed, the deformed surface $S_{i}^{N-1}:=\mathscr{B}_{1}^{N}\left(S_{i}^{N}\right)=\mathscr{B}_{1}^{N}\left(S \cap C_{i}\right)$ lies on the boundary of the hypercube, i.e. within the $(N-1)$-faces of the hypercube. We want it to lie within the $d$-faces of the hypercube, which would imply that it is a stepped surface. To achieve this, we introduce a balloon map

$$
\mathscr{B}_{\alpha}^{N-1, j}:[-1,1]^{N-1} \backslash\{0\} \rightarrow[-1,1]^{N-1} \backslash\{0\}: x \mapsto \begin{cases}\frac{\alpha x}{\|x\|_{\max }} & \text { if }\|x\|_{\max }<\alpha \\ x & \text { if }\|x\|_{\max } \geqslant \alpha\end{cases}
$$

in each of the $(N-1)$-faces $C_{i}^{j}$ of the hypercube $C_{i}$, which pushes the surface into the $(N-2)$-faces. We denote the surface we obtain this way by $S_{i}^{N-2}$. If the surface happens to contain the center of a $(N-1)$-face, we can slightly perturb the surface without affecting the argument. By iterating this procedure, using balloon maps $\mathscr{B}_{\alpha}^{k, j}$ 
in each $k$-face $C_{i}^{j}(N \geqslant k \geqslant d+1)$, we obtain a surface $S_{i}^{d}$ that lies in the $d$-faces (Figs. 5 and 6).

Consider the $(d+1)$-dimensional surface

$$
M_{i}:=\bigcup_{k=d+1}^{N} \bigcup_{\substack{j: C_{i}^{j} \text { is a } \\ k \text {-face of } C_{i}}} \bigcup_{\alpha \in[0,1]} \mathscr{B}_{\alpha}^{k, j}\left(S_{i}^{k} \cap C_{i}^{j}\right)
$$

that is swept out by the consecutive application of the balloon maps to $S_{i}^{N}:=S \cap C_{i}$. Assuming that $\varepsilon$ is small compared to the curvature of $S$, the $(d+1)$-dimensional volume of each of the $\bigcup_{\alpha \in[0,1]} \mathscr{B}_{\alpha}^{k, j}\left(S_{i}^{k} \cap C_{i}^{j}\right)$ is of the order $\varepsilon^{d+1}$. The number of such volumes making up $M_{i}$ only depends on the dimensions $N$ and $d$, not on $\varepsilon$, so the $(d+1)$-dimensional volume $\left|M_{i}\right|$ of $M_{i}$ is of the order $\left|M_{i}\right|=\mathscr{O}\left(\varepsilon^{d+1}\right)$.

Now consider a variation $\mathscr{V}$ with compact support and restrict the surface $S$ to this support. Denote by $\widehat{S}:=\bigcup_{i} S_{i}^{d}$ the stepped surface obtained from $S$ by repeated application of balloon maps in all the hypercubes, and by $M:=\bigcup_{i} M_{i}$ the $(d+1)$ dimensional surface swept out by these balloon maps. The bounary of $M$ consists of $S, \widehat{S}$, and a small strip of area $\mathscr{O}(\varepsilon)$ connecting the boundaries of $S$ and $\widehat{S}$ (the dotted line in Fig. 5). The number of hypercubes intersecting $S$ is of order $\varepsilon^{-d}$, so $|M|=\mathscr{O}\left(\varepsilon^{-d}\right) \mathscr{O}\left(\varepsilon^{d+1}\right)=\mathscr{O}(\varepsilon)$. It follows that

$$
\begin{aligned}
\left|\int_{\widehat{S}} \iota_{\operatorname{pr} \mathscr{V}} \delta \mathscr{L}-\int_{S} \iota_{\operatorname{pr} \mathscr{V}} \delta \mathscr{L}\right| & =\mid \int_{\partial M} \iota_{\operatorname{pr} \mathscr{V} \delta \mathscr{L} \mid+\mathscr{O}(\varepsilon)} \\
& =\left|\int_{M} \mathrm{~d}\left(\iota_{\operatorname{pr} \mathscr{V}} \delta \mathscr{L}\right)\right|+\mathscr{O}(\varepsilon) \rightarrow 0
\end{aligned}
$$

as $\varepsilon \rightarrow 0$. By assumption, $\int_{\widehat{S}} \iota_{\mathrm{pr}} \mathscr{V} \delta \mathscr{L}=0$ for all $\varepsilon$, so the action on $S$ will be stationary as well.
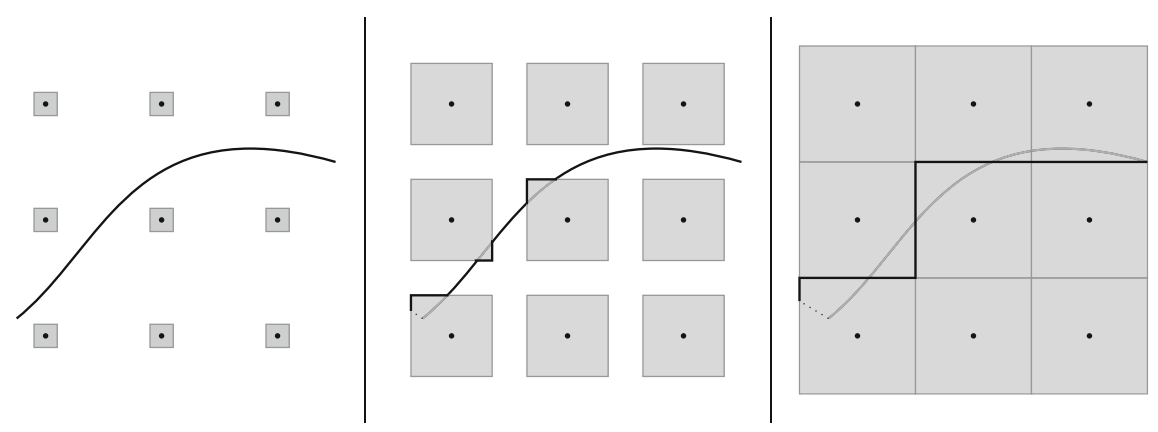

Fig. 5 Balloon maps in nine adjacent squares deforming a curve in $\mathbb{R}^{2}$. From left to right: $\alpha=0.2$, $\alpha=0.7$ and $\alpha=1$ 

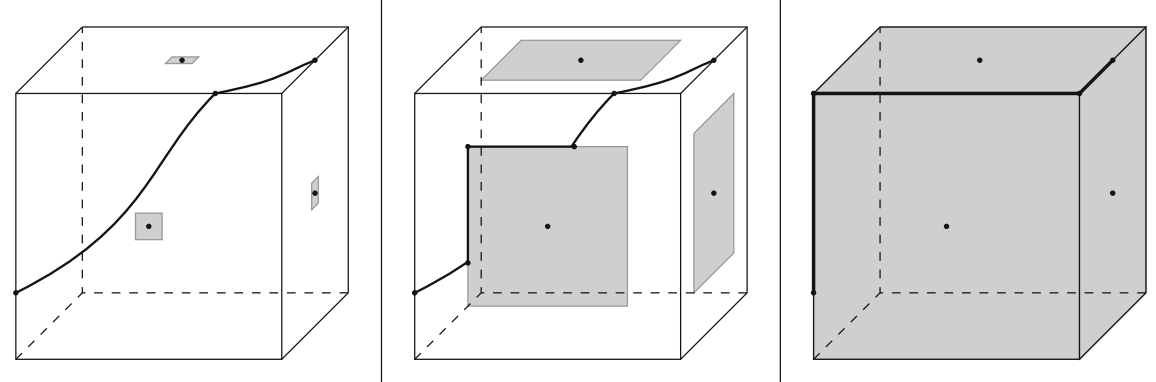

Fig. 6 The second and last iteration for a curve in $\mathbb{R}^{3}$. From left to right: $\alpha=0.1, \alpha=0.6$ and $\alpha=1$

Open Access This chapter is distributed under the terms of the Creative Commons AttributionNoncommercial 2.5 License (http://creativecommons.org/licenses/by-nc/2.5/) which permits any noncommercial use, distribution, and reproduction in any medium, provided the original author(s) and source are credited.

The images or other third party material in this chapter are included in the work's Creative Commons license, unless indicated otherwise in the credit line; if such material is not included in the work's Creative Commons license and the respective action is not permitted by statutory regulation, users will need to obtain permission from the license holder to duplicate, adapt or reproduce the material.

\section{References}

1. Adler, V., Bobenko, A., Suris, Y.: Classification of integrable equations on quad-graphs. the consistency approach. Commun. Math. Phys. 233, 513-543 (2003)

2. Anderson, I.: The variational bicomplex. Preprint (1989)

3. Baxter, R.: Solvable eight-vertex model on an arbitrary planar lattice. Philos. Trans. R. Soc. Lond. 289, 315-346 (1978)

4. Baxter, R.: Free-fermion, checkerboard and Z-invariant lattice models in statistical mechanics. Proc. R. Soc. Lond. A 404, 1-33 (1986)

5. Bazhanov, V., Mangazeev, V., Sergeev, S.: A master solution of the quantum Yang-Baxter equation and classical discrete integrable equations. Adv. Theor. Math. Phys. 16, 65-95 (2012)

6. Bazhanov, V., Mangazeev, V., Sergeev, S.: Faddeev-Volkov solution of the Yang-Baxter equation and discrete conformal symmetry. Nucl. Phys. B 784, 234-258 (2007)

7. Bobenko, A., Mercat, C., Suris, Y.: Linear and nonlinear theories of discrete analytic functions. Intergable structure and isomonodromic Green's function. J. Reine Angew. Math. 583, 117-161 (2005)

8. Bobenko, A., Suris., Y.: Integrable systems on quad-graphs. Intern. Math. Res. Not. 2002(11), 573-611 (2002)

9. Bobenko, A., Suris, Y.: Discrete pluriharmonic functions as solutions of linear pluri-Lagrangian systems. Commun. Math. Phys. 336, 199-215 (2015)

10. Boll, R., Petrera, M., Suris, Y.: What is integrability of discrete variational systems? Proc. R. Soc. A 470, 20130, 550 (2014) 
11. Burstall, F., Ferus, D., Pedit, F., Pinkall, U.: Harmonic tori in symmetric spaces and commuting Hamiltonian systems on loop algebras. Ann. Math. 138, 173-212 (1993)

12. Dickey, L.: Soliton equations and Hamiltonian systems, 2nd edn. World Scientific (2003)

13. Lobb, S., Nijhoff., F.: Lagrangian multiforms and multidimensional consistency. J. Phys. A: Math. Theor. 42, 454,013 (2009)

14. Newell, A.: Solitons in mathematics and physics. SIAM (1985)

15. Nijhoff, F.: Lax pair for the Adler (lattice Krichever-Novikov) system. Phys. Lett. A 297, 49-58 (2002)

16. Noether, E.: Invariante Variationsprobleme. Nachrichten von der Gesellschaft der Wissenschaften zu Göttingen, Math.-Phys. Kl. 235-257 (1918)

17. Ohnita, Y., Valli, G.: Pluriharmonic maps into compact Lie groups and factorization into unitons. Proc. Lond. Math. Soc. 61, 546-570 (1990)

18. Olver, P.: Applications of Lie groups to differential equations, 2nd edn. Springer (1993)

19. Rudin, W.: Function theory in polydiscs. Benjamin (1969)

20. Suris, Y.: Variational formulation of commuting Hamiltonian flows: multi-time Lagrangian 1-forms. J. Geom. Mech. 5, 365-379 (2013)

21. Suris, Y.: Variational symmetries and pluri-Lagrangian systems. In: Dynamical Systems, Number Theory and Applications. A Festschrift in Honor of Armin Leutbecher's 80th Birthday, Hagen, Th., Rupp, F., Scheurle, J. (eds.) World Scientific, pp. 255-266 (2016) 